\title{
EQUICONSISTENCIES AT SUBCOMPACT CARDINALS
}

\author{
ITAY NEEMAN AND JOHN STEEL
}

\begin{abstract}
We present equiconsistency results at the level of subcompact cardinals. Assuming $\mathrm{SBH}_{\delta}$, a special case of the Strategic Branches Hypothesis, we prove that if $\delta$ is a Woodin cardinal and both $\square(\delta)$ and $\square_{\delta}$ fail, then $\delta$ is subcompact in a class inner model. If in addition $\square\left(\delta^{+}\right)$fails, we prove that $\delta$ is $\Pi_{1}^{2}$ subcompact in a class inner model. These results are optimal, and lead to equiconsistencies. As a corollary we also see that assuming the existence of a Woodin cardinal $\delta$ so that $\mathrm{SBH}_{\delta}$ holds, the Proper Forcing Axiom implies the existence of a class inner model with a $\Pi_{1}^{2}$ subcompact cardinal.

Our methods generalize to higher levels of the large cardinal hierarchy, that involve long extenders, and large cardinal axioms up to $\delta$ is $\delta^{+(n)}$ supercompact for all $n<\omega$. We state some results at this level, and indicate how they are proved.
\end{abstract}

MSC 2010: 03E45, 03E55.

Keywords: subcompact cardinals, inner models, long extenders, coherent sequences, square.

We dedicate this paper to Rich Laver, a brilliant mathematician and a kind and generous colleague.

§1. Introduction. We present equiconsistency results at the level of subcompact cardinals. The methods we use extend further, to levels which are interlaced with the axioms $\kappa$ is $\kappa^{+(n)}$ supercompact, for $n<\omega$. The extensions will be carried out in a sequel to this paper, Neeman-Steel [7], but we indicate in this paper some of the main ideas involved.

Our reversals assume iterability for countable substructures of $V$. By a strictly short extender we mean an extender $F$ which maps its critical point strictly above its strength. Let $\mathrm{SBH}_{\delta}$ be the statement that for every countable $H \prec V_{\delta}$, the good player has a winning strategy in the full iteration game of length $\omega_{1}+1$ on the transitive collapse of $H$, with only strictly short extenders allowed, and with the iteration trees restricted to linear compositions of normal, non-overlapping, plus 2 trees. This is a special case of the Strategic Branches Hypothesis of Martin-Steel [4].

Recall that a sequence $\left\langle C_{\alpha} \mid \alpha \in Z \subseteq \delta\right\rangle$ is a coherent sequence on $Z$ if $C_{\alpha}$ is club in $\alpha$ and $\alpha \in \operatorname{Lim}\left(C_{\beta}\right) \cap Z \rightarrow C_{\alpha}=C_{\beta} \cap \alpha$, where $\operatorname{Lim}\left(C_{\beta}\right)$ is the set of limit points of $C_{\beta}$. A thread through a coherent sequence is a club $C \subseteq \delta$, so that for every $\alpha \in \operatorname{Lim}(C) \cap Z, C_{\alpha}=C \cap \alpha$. The statement that there is a coherent sequence on $\delta$ that cannot be threaded is denoted $\square(\delta)$. We will be

This material is based upon work supported by the National Science Foundation under Grants No. DMS-1101204 (Neeman) and DMS-0855692 (Steel), and the Simons Foundation under Simons Fellowship No. 225854 (Neeman). 
concerned mostly with its failure, and will for short say that $\delta$ is threadable if every coherent sequence on $\delta$ has a thread.

Recall that a cardinal $\delta$ is subcompact if for every $A \subseteq H\left(\delta^{+}\right)$there exists $\kappa<\nu<\delta, B \subseteq H(\nu)$, and an elementary embedding of $(H(\nu) ; \kappa, B)$ into $\left(H\left(\delta^{+}\right) ; \delta, A\right)$ with critical point $\kappa$. More generally we say that $\delta$ is $\delta^{+(\alpha)} s u b$ compact, or simply $+(\alpha)$ subcompact, if the above holds with $\delta^{+}$replaced by $\delta^{+(\alpha)}$. We allowed freedom for $\nu$ in the definition of $+(\alpha)$ subcompactness to make it general, but of course if $\alpha<\delta$ then by elementarity $\nu$ must be $\kappa^{+(\alpha)}$. By standard arguments these large cardinal notions are interlaced with supercompactness, in the sense that (assuming GCH above $\delta$ ), $\delta$ is $+(\alpha+2)$ subcompact $\rightarrow \delta$ is $\delta^{+(\alpha)}$ supercompact $\rightarrow \delta$ is $+(\alpha)$ subcompact.

The following lemma gives well known consequences of subcompactness:

Lemma 1.1. Suppose $\delta$ is subcompact. Then $\delta$ is a Woodin cardinal, $\delta$ is weakly compact which in particular implies $\delta$ is threadable, and $\square_{\delta}$ fails.

We prove a reversal of this lemma, assuming iterability:

Theorem 1.2. Assume $\mathrm{SBH}_{\delta}$. Suppose that $\delta$ is a Woodin cardinal, $\delta$ is threadable, and $\square_{\delta}$ fails. Then $\delta$ is subcompact in a class inner model.

The model we construct in the proof of Theorem 1.2 is a fine structural model whose countable elementary substructures are $\omega_{1}+1$ iterable. By Theorem 3.3 of Steel [13] models of this kind satisfy the Unique Branches Hypothesis (UBH) for normal, non-overlapping, plus 2 trees. Hence we obtain the next corollary as an immediate consequence.

COROLlary 1.3. The following are equiconsistent:

1. $\mathrm{SBH}_{\delta}, \delta$ is a Woodin cardinal, $\delta$ is weakly compact, and $\square_{\delta}$ fails.

2. $\mathrm{SBH}_{\delta}, \delta$ is a Woodin cardinal, $\delta$ is threadable, and $\square_{\delta}$ fails.

3. $\delta$ is subcompact and UBH (for normal non-overlapping plus 2 trees) holds.

4. $\delta$ is subcompact and $\mathrm{SBH}_{\delta}$ holds.

By a $\Pi_{1}^{1}$ formula we mean a formula $\varphi$ of the form $(\forall X) \psi$ where $X$ is a second order variable and all quantifiers in $\psi$ are over first order variables. When interpreted over $H\left(\delta^{+}\right)$, the second order variables range over subsets of $H\left(\delta^{+}\right)$ and the first order variables range over elements of $H\left(\delta^{+}\right)$. Recall that a cardinal $\delta$ is $\Pi_{1}^{2}$ subcompact if for every $A \subseteq H\left(\delta^{+}\right)$and every $\Pi_{1}^{1}$ formula $\varphi$ that holds of $A$ over $H\left(\delta^{+}\right)$, there exists $\kappa<\delta$ and $B \subseteq H\left(\kappa^{+}\right)$witnessing the subcompactness of $\delta$ for $A$, with the additional property that $\varphi$ holds of $B$ over $H\left(\kappa^{+}\right) . \Pi_{1}^{1}$ formulas interpreted over $H\left(\delta^{+}\right)$are equivalent to $\Pi_{1}^{2}$ formulas interpreted over $H(\delta)$. $\Pi_{1}^{2}$ subcompactness combines reflection for these formulas with subcompactness.

The following lemma about consequences of $\Pi_{1}^{2}$ subcompactness for threading is standard. The conclusion of the lemma implies in particular that $\square_{\delta}$ fails, since any $\square_{\delta}$ sequence is a threadless coherent sequence on $\delta^{+}$. But the lemma gives more. It applies to any coherent sequence, while $\square_{\delta}$ sequences $\left\langle C_{\alpha} \mid \alpha<\delta^{+}\right\rangle$ have the restricting property that the ordertype of $C_{\alpha}$ is $\leq \delta$.

We will see in Theorem 4.6 that in weakly iterable premice, the conclusion of Lemma 1.4 precisely characterizes the $\Pi_{1}^{2}$ subcompact cardinals. This fact is closely related to work of Kypriotakis-Zeman [3]. 
Lemma 1.4. Let $\delta$ be $\Pi_{1}^{2}$ subcompact. Then $\delta^{+}$is threadable.

Proof. Suppose for contradiction $\vec{C}=\left\langle C_{\alpha} \mid \alpha<\delta^{+}\right\rangle$is a coherent sequence without a thread. Note that the inexistence of a thread is a $\Pi_{1}^{1}$ statement about the sequence. Using $\Pi_{1}^{2}$ subcompactness, find $\kappa<\delta$, a coherent sequence $\vec{D}=\left\langle D_{\alpha} \mid \alpha<\kappa^{+}\right\rangle$on $\kappa^{+}$which does not have a thread, and elementary $\pi:\left(H\left(\kappa^{+}\right) ; \kappa, \vec{D}\right) \rightarrow\left(H\left(\delta^{+}\right) ; \delta, \vec{C}\right)$ with critical point $\kappa$. But now let $\alpha=\sup \left(\pi^{\prime \prime} \kappa^{+}\right)$and note that $\pi^{-1 \prime \prime} C_{\alpha}$ generates a thread through $\vec{D}$.

The following theorem reverses the consequences of $\Pi_{1}^{2}$ subcompactness given by Lemmas 1.1 and 1.4. The subsequent corollary gives immediate consequences that parallel Corollary 1.3.

TheOREM 1.5. Assume $\mathrm{SBH}_{\delta}$. Suppose that $\delta$ is a Woodin cardinal, $\delta$ is threadable, and $\delta^{+}$is threadable. Then $\delta$ is $\Pi_{1}^{2}$ subcompact in a class inner model.

COROllary 1.6. The following are equiconsistent:

1. $\mathrm{SBH}_{\delta}, \delta$ is a Woodin cardinal, $\delta$ is weakly compact, and $\delta^{+}$is threadable.

2. $\mathrm{SBH}_{\delta}, \delta$ is a Woodin cardinal, $\delta$ is threadable, and $\delta^{+}$is threadable.

3. $\delta$ is $\Pi_{1}^{2}$ subcompact and $\mathrm{UBH}$ (for normal non-overlapping plus 2 trees) holds.

4. $\delta$ is $\Pi_{1}^{2}$ subcompact and $\mathrm{SBH}_{\delta}$ holds.

Since PFA, the proper forcing axiom, implies that every regular $\lambda \geq \omega_{2}$ is threadable, the following is another immediate corollary of Theorem 1.5. It gives substantially more than any strength previously extracted from PFA, but under the assumption that there exists a Woodin cardinal $\delta$ and $\mathrm{SBH}_{\delta}$ holds.

Corollary 1.7. Suppose that $\delta$ is a Woodin cardinal and $\mathrm{SBH}_{\delta}$ holds. Then PFA implies that there is a class inner model with a $\Pi_{1}^{2}$ subcompact cardinal.

Results along the lines of Theorem 1.2 were obtained in Jensen-SchimmerlingSchindler-Steel [2], but using an iterability assumption on the constructed fine structural model that is not a known consequence of iterability of $V$. The structure of our proof is similar to that in [2], but we replace the partial backgrounded construction there with a fully backgrounded construction, and this is the reason we can work under standard assumptions of iterability for $V$, and consequently can get actual equiconsistencies in Corollaries 1.3 and 1.6.

Normally a full backgrounded construction would not achieve the kind of weak covering needed for results such as Theorem 1.2 or the results in [2]. Two components in our argument take care of this, allowing us to obtain the necessary weak covering. One is a modification to the background condition, requiring extenders placed on the sequence to embed into coarse extenders on $V$, rather than be equal to restrictions of coarse extenders on $V$. Another is a use of the Woodin cardinal, to obtain a rich collection of coarse extenders into which we can embed. The latter relies on some ideas that trace back to Mitchell-Schindler [5], and has precursors in the proof of Lemma 11.1 of Steel [15] for universality of fully backgrounded constructions up to a Woodin cardinal, and in an argument of Steel (previously published only in the context of HOD-mice, in Sargsyan 
[9, Lemma 5.2]) obtaining high cofinality of the stack over a fully backgrounded construction up to a Woodin cardinal.

Our background condition is given precisely in Section 2. Then in Section 3 we present the necessary results from [2]. The proof of Theorem 1.2 is given in Section 4, and the use of the two components described above is in Claim 4.1, where we argue that the stack over our fully backgrounded construction up to $\delta$ must have height of cofinality at least $\delta$.

As mentioned above our methods generalize to levels of the large cardinal hierarchy that involve long extenders. These generalizations still use only strictly short extender iterability assumptions. One example of the results we get is Theorem 1.9 below. The generalizations involve a theory of fine structure for long extenders that is outside the scope of this paper. But we give some of the main ideas for the generalizations here, in Section 5 .

Let Strong $(A, \delta)$ denote the set $\{\kappa<\delta \mid \kappa$ is $<\delta$ strong relative to $A\}$. Let $\mathcal{F}_{W}(\delta)$ denote the Woodin filter on $\delta$, namely the filter generated by the sets Strong $(A, \delta)$ for $A \subseteq \delta$. The filter is non-trivial iff $\delta$ is a Woodin cardinal.

For a weakly compact cardinal $\delta$ and $Z \subseteq \delta$, we say that the weak compactness of $\delta$ can be witnessed by partial measures concentrating on $Z$ if for every $U$ of size $\delta$, there is a $<\delta$ complete, normal relative to functions in $U$, non-trivial, partial measure $\mu$ on $\delta$, with $U \cap \mathcal{P}(\delta) \subseteq \operatorname{dom}(\mu)$, and $\mu(Z)=1$. The important connection with the set $Z$ is that $\delta \in i_{\mu}(Z)$, where $i_{\mu}$ is the ultrapower embedding of $U$ by $\mu$, assuming $Z \in U$ and $U$ is rich enough that standard properties of the ultrapower construction by total normal measures apply.

The results we state below involve indestructibility under collapses, of weak compactness by measures concentrating on arbitrary sets in the Woodin filter. Without the indestructibility, the existence of measures concentrating on sets in the Woodin filter is substantially below the large cardinals we deal with here, though it still has non-trivial strength. For example it is easy to check that the following statements are strictly descending in the large cardinal hierarchy, meaning that below each cardinal witnessing one of the statements there are many cardinals witnessing the subsequent statements: $\kappa$ is superstrong; there is a (total) normal measure extending $\mathcal{F}_{W}(\kappa) ; \kappa$ is Shelah; for every $Z \in \mathcal{F}_{W}(\kappa)$ there is a (total) normal measure concentrating on $Z ; \kappa$ is weakly compact and for every $Z \in \mathcal{F}_{W}(\kappa)$ this can be witnessed by partial measures concentrating on $Z ; \kappa$ is a measurable Woodin cardinal.

One needs substantially more to add indestructibility to weak compactness of $\delta$ by measures concentrating on arbitrary sets in $\mathcal{F}_{W}(\delta)$. The following results show that indestructibility through $\operatorname{Col}\left(\delta, \delta^{+(n)}\right)$ for all $n<\omega$ corresponds to $\delta^{+(n)}$ supercompactness for all $n<\omega$. The forward direction is standard. It is the reversal in Theorem 1.9 that is new.

LEMma 1.8. Suppose that for all $n<\omega, \delta$ is $\delta^{+(n)}$ supercompact, and $2^{\delta^{+(n)}}=$ $\delta^{+(n+1)}$. Then there is a forcing extension which satisfies the following: for all $n<\omega, \Vdash_{\mathrm{Col}\left(\delta, \delta^{+(n)}\right)}$ " $\delta$ is weakly compact and for every set $Z$ in the Woodin filter for $\delta$ the weak compactness of $\delta$ can be witnessed by partial measures concentrating on $Z$ ". 
PROOF. This is a standard indestructibility argument, and in fact one can get much more, for example reaching an extension that satisfies: for all $n<\omega$, it is forced in $\operatorname{Col}\left(\delta, \delta^{+(n)}\right)$ that $\delta$ is $2^{\delta}$ supercompact.

THEOREM 1.9. Assume $\mathrm{SBH}_{\delta}$. Suppose that for all $n<\omega, \Vdash_{\mathrm{Col}\left(\delta, \delta^{+(n)}\right)}$ " $\delta$ is weakly compact and for every set $Z$ in the Woodin filter for $\delta$ the weak compactness of $\delta$ can be witnessed using partial measures concentrating on $Z$ ". Then there is a class inner model in which $\mathrm{GCH}$ holds and for all $n<\omega, \delta$ is $\delta^{+(n)}$ supercompact.

Fine structural inner models for large cardinals at the level of Theorem 1.9 were first developed by Woodin, see for example Woodin [16]. Neeman-Steel [7] use a different hierarchy, developed by the authors. Some key ideas for this development are due to earlier work of Steel and of Woodin; we say more on this in Remark 5.1. After hearing of the hierarchy Woodin showed that a close variant of it is interpreted in his own hierarchy, and it is natural to expect at this stage that the exact hierarchy of [7] may be interpretable in Woodin's hierarchy. For details on this see the revised hierarchy in Woodin [16, Chapter 12].

We conjecture that the results of Schimmerling-Zeman $[11,12]$ on failure of $\square$ in inner models generalize to long extender hierarchies. Assuming the natural generalization to the hierarchy of [7], Theorem 1.9 has level-by-level refinements, that lead to generalizations of Theorem 1.2 , with $\square_{\delta}$ replaced by $\square_{\delta+(n)}$, threading of coherent sequences on $\delta$ replaced by threading of coherent sequences on sets in the Woodin filter for $\delta$ in $V^{\operatorname{Col}\left(\delta, \delta^{+(n)}\right)}$, and subcompactness replaced with $+(n+1)$ subcompactness. This provides an exact match, as all the assumptions can be forced assuming $+(n+1)$ subcompactness, and leads to equiconsistencies generalizing Corollary 1.3.

We do not prove Theorem 1.9 in this paper, but Section 5 has a construction that allows us to explain some of the main ideas in the proof of the theorem without getting into details of the fine structural models used. A full proof will appear in [7].

$\S 2$. The main backgrounded construction. We follow the fine structure conventions of Andretta-Neeman-Steel [1]. In particular this means that we use Jensen indexing, and that to each premouse $\mathcal{M}$ we have associated $k(\mathcal{M}) \leq \omega$. $\mathcal{M}$ is always $k(\mathcal{M})$ sound. The projectum of $\mathcal{M}$ is the $k(\mathcal{M})+1$ projectum. The standard parameter of $\mathcal{M}$ is the part of the $k(\mathcal{M})+1$ standard parameter of $\mathcal{M}$ below the $k(\mathcal{M})$ projectum. Elementarity for maps on $\mathcal{M}$ and hulls in $\mathcal{M}$ is $r \Sigma_{k(\mathcal{M})+1}$ elementarity, equivalently $\Sigma_{1}^{(k(\mathcal{M}))}$ elementarity. Levels of our premice are indexed by pairs $\langle\mu, l\rangle$, and $\mathcal{M} \|\langle\mu, l\rangle$ is a premouse with $k(\mathcal{M} \|\langle\mu, l\rangle)=l$. Andretta-Neeman-Steel [1] avoids superstrong extenders, but the notions there apply also to premice with superstrong extenders; the only additional point to note is that if the top extender of a premouse $\mathcal{M}$ has a largest cut point, then the index of the restriction of the top extender to its largest cut point is added as a constant in the premouse structure.

We use $\mathcal{M} \uparrow \mu$ to denote the premouse obtained from $\mathcal{M} \|\langle\mu, 0\rangle$ by removing its top extender predicate (if there is one). In the case that $\omega \cdot \mu$ is the ordinal 


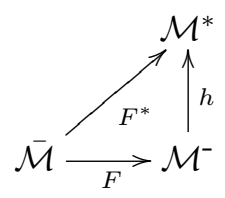

Diagram 1. The background condition

height of $\mathcal{M}$, so that $\mathcal{M}\lceil\mu$ has the same elements as $\mathcal{M}$ but no top extender, we write $\mathcal{M}^{-}$for $\mathcal{M} \uparrow \mu$.

Section 2 of [1] presents the $K^{c}$ partially backgrounded construction under these conventions. Our own construction is the same, only replacing the notion of certificate in Definition 2.20 of [1] with the following:

Definition 2.1. Let $\mathcal{M}$ be an active premouse with a top extender $F$. Let $\kappa=\operatorname{crit}(F)$, and let $\lambda=F(\operatorname{crit}(F))$. Then $\mathcal{M}$ is certifiable iff there exists a strictly short extender $F^{*}$ over $V$, and an embedding $h$, so that:

1. $\operatorname{crit}\left(F^{*}\right)=\kappa$, the strength of $F^{*}$ is an inaccessible cardinal, say $\eta$, and $\mathcal{M} \in V_{\eta}$.

2. Let $\overline{\mathcal{M}}=\mathcal{M} \uparrow\left(\kappa^{+}\right)^{\mathcal{M}}$, so that $F$ is an embedding of $\overline{\mathcal{M}}$ into $\mathcal{M}^{-}$. Let $i_{F^{*}}$ be the ultrapower embedding of $V$ by $F^{*}$, and let $\mathcal{M}^{*}=i_{F^{*}}(\overline{\mathcal{M}})$. Then $h$ is an elementary embedding from $\mathcal{M}^{-}$into $\mathcal{M}^{*}$ with $\operatorname{crit}(h) \geq \lambda$, and $h \circ F=i_{F^{*}}\lceil\overline{\mathcal{M}}$.

We refer to the pair $\left\langle F^{*}, h\right\rangle$ as a certificate for $\mathcal{M}$, and occasionally also as a certificate for $F$.

We phrased Definition 2.1 in a way that will generalize to situations where $F$ is a long extender. In the current context, where the generators of $F$ are contained in $\lambda=F(\operatorname{crit}(F))$, the definition can be phrased without reference to $h$, requiring simply that $F(A)=i_{F^{*}}(A) \cap \lambda$ for all $A \in \overline{\mathcal{M}}$. (To see that this phrasing implies the one in the definition, assuming $F$ has no long generators, take $h$ to be the function $F(g)(a) \mapsto i_{F^{*}}(g)(a)$ for finite $a \subseteq \lambda$ and functions $g \in \overline{\mathcal{M}}$ with $\operatorname{dom}(g)=\kappa^{<\omega}$.) The difference between this condition, and the standard certifiability condition for a full background construction using Jensen indexing, is that we allow $i_{F^{*}}(\kappa)>\lambda$.

Fix a cardinal $\delta$. Let $\mathcal{M}_{\nu, k}$ for $\langle\nu, k\rangle \leq_{\text {Lex }}\langle\delta, 0\rangle$ be given by the construction in Section 2 of Andretta-Neeman-Steel [1], only replacing the certifiability requirement in cases $1 \mathrm{a}$ and $1 \mathrm{~b}$ of the construction with certifiability in the sense of the current Definition 2.1, via a certificate in $V_{\delta}$.

As explained in Section 2 of [1], the construction of the models $\mathcal{M}_{\nu, k}$ requires certain properties that can be proved inductively provided that the levels constructed are sufficiently iterable. The amount of iterability needed for the construction to succeed in general, with no smallness conditions on the large cardinals allowed in the models, is given by condition $(\dagger)_{\nu, k}$ of Theorem 2.21 in [1]. Using the results of Neeman-Steel [8], the condition holds if, for all sufficiently closed countable $H \prec V_{\delta}$ with $\mathcal{M}_{\nu, k} \in H$, the good player has a winning strategy in the length $\omega_{1}+1$ iteration game (for countable linear compositions 
of normal, maximal, non-overlapping iteration trees in the sense of [1]) on the image of $\mathcal{M}_{\nu, k}$ under the transitive collapse map for $H$. This in turn would be a direct consequence of $\mathrm{SBH}_{\delta}$, provided that the good player's role in the iteration game on $\mathcal{M}_{\nu, k}$ can be reduced to her role in the (coarse) iteration game on $V_{\delta}$. Such a reduction can be obtained from a round by round conversion of (normal, maximal, non-overlapping) iteration trees on $\mathcal{M}_{\nu, k}$ to iteration trees on $V_{\delta}$, so that each model of the tree on $\mathcal{M}_{\nu, k}$ embeds elementarily into a level of the construction in a model of the tree on $V_{\delta}$. We continue to describe a conversion that does this. It is similar to the conversion used for the same purpose in Mitchell-Steel [6], but with a slight change to the generation of embeddings from the fine structural models into the coarse models, to account for the maps $h$ allowed in Definition 2.1.

Define the resurrection at $\langle\nu, k\rangle$ exactly as in Section 2 of Andretta-NeemanSteel [1]. The resurrection is a pair of functions $\operatorname{Res}_{\nu, k}$ and $\sigma_{\nu, k}$ which relate initial segments of $\mathcal{M}_{\nu, k}$ to the stage in the construction where they appeared. For each initial segment $\mathcal{N}$ of $\mathcal{M}_{\nu, k}, \operatorname{Res}_{\nu, k}[\mathcal{N}]$ is a stage $\langle\eta, l\rangle \leq\langle\nu, k\rangle$, and $\sigma_{\nu, k}[\mathcal{N}]$ is an elementary embedding of $\mathcal{N}$ into $\mathcal{M}_{\eta, l}$. The definition is by induction on the lexicographic order for pairs $\langle\nu, k\rangle$. We refer the reader to [1] for the details, and only note the following points on agreements between the maps: If $\mathcal{N} \unlhd \mathcal{M}_{\nu, k}$, and $\mathcal{N} \unlhd \mathcal{N}^{\prime} \triangleleft \mathcal{M}_{\nu, k} \rightarrow \rho\left(\mathcal{N}^{\prime}\right) \geq \gamma$, then $\sigma_{\langle\nu, k\rangle}[\mathcal{N}] \mid \gamma=i d$. Further, if $\mathcal{N} \unlhd \mathcal{N}^{*} \unlhd \mathcal{M}_{\nu, k}$, and $\mathcal{N} \unlhd \mathcal{N}^{\prime} \triangleleft \mathcal{N}^{*} \rightarrow \rho\left(\mathcal{N}^{\prime}\right) \geq \gamma$, then $\sigma_{\langle\nu, k\rangle}[\mathcal{N}] \mid \gamma=\sigma_{\langle\nu, k\rangle}\left[\mathcal{N}^{*}\right]\lceil\gamma$.

Definition 2.2. Let $\mathcal{T}$ be a normal, maximal, non-overlapping iteration tree on $\mathcal{M}_{\nu, k}$. A conversion system for $\mathcal{T}$ is an iteration tree $\mathcal{T}^{*}$ on $V$, indices $\left\langle\eta_{\xi}, l_{\xi}\right\rangle$ for $\xi<\operatorname{lh}(\mathcal{T})$, and maps $\pi_{\xi}$ for $\xi<\operatorname{lh}(\mathcal{T})$, so that, using $\mathcal{P}_{\xi}, i_{\xi, \zeta}, F_{\xi}$, and $P_{\xi}^{*}$, $i_{\xi, \zeta}^{*}$, and $F_{\xi}^{*}$ to denote the models, embeddings, and extenders of $\mathcal{T}$ and $\mathcal{T}^{*}$ respectively:

1. $\left\langle\eta_{\xi}, l_{\xi}\right\rangle$ belongs to $P_{\xi}^{*}$, and $\pi_{\xi}: \mathcal{P}_{\xi} \rightarrow\left(\mathcal{M}_{\eta_{\xi}, l_{\xi}}\right)^{P_{\xi}^{*}}$ is elementary.

2. $\mathcal{T}$ and $\mathcal{T}^{*}$ have the same tree order.

3. Suppose $\xi<_{\mathcal{T}} \zeta$ and there are no truncations on $[\xi, \zeta)_{\mathcal{T}}$. Then $\left\langle\eta_{\zeta}, l_{\zeta}\right\rangle=$ $i_{\xi, \zeta}^{*}\left(\left\langle\eta_{\xi}, l_{\xi}\right\rangle\right)$ and $\pi_{\zeta} \circ i_{\xi, \zeta}=i_{\xi, \zeta}^{*} \circ \pi_{\xi}$.

4. Suppose $\xi$ is the $<_{\mathcal{T}}$ predecessor of $\epsilon+1$, is $\xi$ is a truncation on the branch to $\epsilon+1$, and the truncation is to the initial segment $\overline{\mathcal{P}}$ of $\mathcal{P}_{\xi}$. Then $\left\langle\eta_{\epsilon+1}, l_{\epsilon+1}\right\rangle=i_{\xi, \epsilon+1}^{*}\left(\operatorname{Res}_{\eta_{\xi}, l_{\xi}}^{P_{\xi}^{*}}\left[\pi_{\xi}(\overline{\mathcal{P}})\right]\right)$.

5. Let $\lambda_{\xi}=F_{\xi}\left(\operatorname{crit}\left(F_{\xi}\right)\right)$. Let $\alpha_{\xi}$ be the index of $F_{\xi}$ in $\mathcal{P}_{\xi}$, and let $\sigma_{\xi}$ be the resurrection map $\sigma_{\eta_{\xi}, l_{\xi}}^{P_{\xi}^{*}}\left[\pi_{\xi}\left(\mathcal{P}_{\xi} \|\left\langle\alpha_{\xi}, 0\right\rangle\right)\right]$. Then for $\xi<\zeta, \pi_{\zeta}\left\lceil\lambda_{\xi}=\right.$ $\sigma_{\xi} \circ \pi_{\xi} \backslash \lambda_{\xi}$, and $P_{\xi}^{*}$ and $P_{\zeta}^{*}$ agree up to $\sup \left(\sigma_{\xi} \circ \pi_{\xi}\right)^{\prime \prime} \lambda_{\xi}$.

Definition 2.2 gives a standard association between trees on levels of a fully backgrounded inner model construction and trees on $V$, for a construction using Jensen indexing.

It is clear that a union of increasing conversion systems for increasing trees $\mathcal{T}_{\alpha}$ is a conversion system for $\bigcup \mathcal{T}_{\alpha}$. It is also clear that a conversion system through a tree $\mathcal{T}$ of limit length can be extended (uniquely) to the tree resulting from the addition of any given cofinal branch through $\mathcal{T}$. The next claim shows that conversion systems can also be extended in the successor case. 
Claim 2.3. Let $\mathcal{T}$ and $\mathcal{T}^{\prime}$ be normal, maximal, non-overlapping trees, of length $\epsilon+1$ and $\epsilon+2$ respectively with $\mathcal{T}^{\prime}$ extending $\mathcal{T}$. Then any conversion system for $\mathcal{T}$ extends to a conversion system for $\mathcal{T}^{\prime}$.

Proof. We use the notation of Definition 2.2. $\mathcal{P}_{\epsilon}$ is the last model of $\mathcal{T}$. $F_{\epsilon} \in \mathcal{P}_{\epsilon}$ is used to form $\mathcal{P}_{\epsilon+1}$, the last model of $\mathcal{T}^{\prime}$. Let $\xi$ be the predecessor of $\epsilon+1$ in $\left\langle\mathcal{T}^{\prime}\right.$. Since $\mathcal{T}^{\prime}$ is normal and non-overlapping, $\xi$ is least so that $\operatorname{crit}\left(F_{\epsilon}\right)<\lambda_{\xi}$.

$\alpha_{\epsilon}$ is the index of $F_{\epsilon}$ in $\mathcal{P}_{\epsilon}$. The resurrection map $\sigma_{\epsilon}$ embeds $\pi_{\epsilon}\left(\mathcal{P}_{\epsilon} \|\left\langle\alpha_{\epsilon}, 0\right\rangle\right)$ into a level of our backgrounded construction in $P_{\epsilon}^{*}$, and maps $\pi_{\epsilon}\left(F_{\epsilon}\right)$ to a top extender of this level, call it $\hat{F}_{\epsilon}$. By the certifiability requirements of our construction, there is a certificate $\left\langle F_{\epsilon}^{*}, h\right\rangle$ for $\hat{F}_{\epsilon}$ in $V_{i_{0, \epsilon}^{*}}^{P_{\epsilon}^{*}}(\delta)$.

Extend $\mathcal{T}^{*}$ by letting $P_{\epsilon+1}^{*}=\operatorname{Ult}_{0}\left(P_{\xi}^{*}, F_{\epsilon}^{*}\right)$. If $\xi<\epsilon$ this extension requires some agreement between $P_{\xi}^{*}$ and $P_{\epsilon}^{*}$. In this case there are no strict initial segments of $\mathcal{P}_{\epsilon}$ which project across $\lambda_{\xi}$. Since $\operatorname{crit}\left(F_{\epsilon}\right)<\lambda_{\xi}<\alpha_{\epsilon}$ it follows that there are no strict initial segments of $\mathcal{P}_{\epsilon}$ at or above $\mathcal{P}_{\epsilon} \|\left\langle\alpha_{\epsilon}, 0\right\rangle$ which project to $\operatorname{crit}\left(F_{\epsilon}\right)$. So $\sigma_{\epsilon}\left\lceil\pi_{\epsilon}\left(\operatorname{crit}\left(F_{\epsilon}\right)\right)+1=i d\right.$, and hence $\operatorname{crit}\left(F_{\epsilon}^{*}\right)=\operatorname{crit}\left(\hat{F}_{\epsilon}\right)=$ $\sigma_{\epsilon}\left(\pi_{\epsilon}\left(\operatorname{crit}\left(F_{\epsilon}\right)\right)\right)=\pi_{\epsilon}\left(\operatorname{crit}\left(F_{\epsilon}\right)\right)$. By condition (5) of Definition 2.2 and since $\operatorname{crit}\left(F_{\epsilon}\right)<\lambda_{\xi}$ it then follows that $P_{\xi}^{*}$ and $P_{\epsilon}^{*}$ agree past $\operatorname{crit}\left(F_{\epsilon}^{*}\right)$, as required for extending $\mathcal{T}^{*}$.

Suppose $\mathcal{P}_{\epsilon+1}$ is formed without a truncation. Then, letting $\mathfrak{D}\left(\mathcal{P}_{\xi}\right)$ denote the master structure for $\mathcal{P}_{\xi}$ (called the true domain in [1]), $\mathcal{P}_{\epsilon+1}$ is determined uniquely by the fact that $\mathfrak{D}\left(\mathcal{P}_{\epsilon+1}\right)=\operatorname{Ult}_{0}\left(\mathfrak{D}\left(\mathcal{P}_{\xi}\right), F_{\epsilon}\right)$, and $i_{\xi, \epsilon+1}: \mathcal{P}_{\xi} \rightarrow \mathcal{P}_{\epsilon+1}$ is the embedding induced by the ultrapower embedding between the master structures. To determine $\pi_{\epsilon+1}$ it is enough to define it on $\mathfrak{D}\left(\mathcal{P}_{\epsilon+1}\right)$.

Each element of $\mathfrak{D}\left(\mathcal{P}_{\epsilon+1}\right)$ has the form $i_{\xi, \epsilon+1}(f)(a)$ for some function $f \in$ $\mathfrak{D}\left(\mathcal{P}_{\xi}\right)$ and finite $a \subseteq \lambda_{\epsilon}$. Set $\pi_{\epsilon+1}\left(i_{\xi, \epsilon+1}(f)(a)\right)=\left(i_{\xi, \epsilon+1}^{*} \circ \pi_{\xi}(f)\right)\left(h \circ \sigma_{\epsilon} \circ \pi_{\epsilon}(a)\right)$. For this to be well defined requires that for any $X \subseteq \operatorname{crit}\left(F_{\epsilon}\right)$ in $\mathcal{P}_{\xi}$, and any $\beta \in \lambda_{\epsilon}, \beta \in F_{\epsilon}(X)$ iff $h \circ \sigma_{\epsilon} \circ \pi_{\epsilon}(\beta) \in F_{\epsilon}^{*} \circ \pi_{\xi}(X)$. This equivalence can be obtained as follows:

$$
\begin{aligned}
\beta \in F_{\epsilon}(X) & \Longleftrightarrow \sigma_{\epsilon} \circ \pi_{\epsilon}(\beta) \in\left(\sigma_{\epsilon} \circ \pi_{\epsilon}\left(F_{\epsilon}\right)\right)\left(\sigma_{\epsilon} \circ \pi_{\epsilon}(X)\right) \\
& \Longleftrightarrow \sigma_{\epsilon} \circ \pi_{\epsilon}(\beta) \in \hat{F}_{\epsilon}\left(\sigma_{\epsilon} \circ \pi_{\epsilon}(X)\right) \\
& \Longleftrightarrow \sigma_{\epsilon} \circ \pi_{\epsilon}(\beta) \in \hat{F}_{\epsilon}\left(\pi_{\epsilon}(X)\right) \\
& \Longleftrightarrow h \circ \sigma_{\epsilon} \circ \pi_{\epsilon}(\beta) \in h \circ \hat{F}_{\epsilon}\left(\pi_{\epsilon}(X)\right) \\
& \Longleftrightarrow h \circ \sigma_{\epsilon} \circ \pi_{\epsilon}(\beta) \in F_{\epsilon}^{*}\left(\pi_{\epsilon}(X)\right) \\
& \Longleftrightarrow h \circ \sigma_{\epsilon} \circ \pi_{\epsilon}(\beta) \in F_{\epsilon}^{*}\left(\sigma_{\xi} \circ \pi_{\xi}(X)\right) \\
& \Longleftrightarrow h \circ \sigma_{\epsilon} \circ \pi_{\epsilon}(\beta) \in F_{\epsilon}^{*}\left(\pi_{\xi}(X)\right) .
\end{aligned}
$$

The equivalence (3) holds because in the case of no truncation, $\sigma_{\epsilon}$ is the identity on subsets of $\pi_{\epsilon}\left(\operatorname{crit}\left(F_{\epsilon}\right)\right)$, by properties of the resurrection maps listed above and the fact that no strict initial segments of $\mathcal{P}_{\epsilon}$ at or above $\mathcal{P}_{\epsilon} \|\left\langle\alpha_{\epsilon}, 0\right\rangle$ project to $\operatorname{crit}\left(F_{\epsilon}\right)$. The equivalence (6) uses the agreement between $\sigma_{\xi} \circ \pi_{\xi}$ and $\pi_{\epsilon}$ given by condition (5) of Definition 2.2 if $\xi<\epsilon$, and if $\xi=\epsilon$ it simply reverts to the argument of $\hat{F}_{\epsilon}$ in line (2). The equivalence (7) uses the fact that we are working in a case without truncations, to see that $\sigma_{\xi}$ is the identity on 
subsets of $\pi_{\xi}\left(\operatorname{crit}\left(F_{\epsilon}\right)\right)$. It is for the equivalences (4) and (5) that we use the properties of the certificate $\left\langle F_{\epsilon}^{*}, h\right\rangle$, specifically the elementarity of $h$ and the equality $\left(h \circ \hat{F}_{\epsilon}\right)(X)=F_{\epsilon}^{*}(X)$ for $X \subseteq \operatorname{crit}\left(\hat{F}_{\epsilon}\right)$ in the domain of $\hat{F}_{\epsilon}$, given by condition (2) of Definition 2.1.

It is easy to check directly from our definition of $\pi_{\epsilon+1}$ above, using $f=i d$, that for every $\beta<\lambda_{\epsilon}, \pi_{\epsilon+1}(\beta)=h \circ \sigma_{\epsilon} \circ \pi_{\epsilon}(\beta)$. By Definition 2.1, $\operatorname{crit}(h) \geq$ $\hat{F}_{\epsilon}\left(\operatorname{crit}\left(\hat{F}_{\epsilon}\right)\right)=\sigma_{\epsilon} \circ \pi_{\epsilon}\left(\lambda_{\epsilon}\right)$. So $\pi_{\epsilon+1}\left\lceil\lambda_{\epsilon}=\sigma_{\epsilon} \circ \pi_{\epsilon} \uparrow \lambda_{\epsilon}\right.$. Moreover since $F_{\epsilon}^{*}$ is $\eta$ strong for some $\eta>\hat{F}_{\epsilon}\left(\operatorname{crit}\left(\hat{F}_{\epsilon}\right)\right), P_{\epsilon+1}^{*}$ and $P_{\epsilon}$ agree past $\sigma_{\epsilon} \circ \pi_{\epsilon}\left(\lambda_{\epsilon}\right)$. It follows from these two facts that condition (5) of Definition 2.2 is preserved, and this completes the proof of the claim in case $\mathcal{P}_{\epsilon+1}$ is formed without a truncation.

Suppose next that $\mathcal{P}_{\epsilon+1}$ is formed with a truncation. Let $\overline{\mathcal{P}}$ be the first initial segment of $\mathcal{P}_{\xi}$ at or above $\mathcal{P}_{\xi} \|\left\langle\alpha_{\xi}, 0\right\rangle$ which projects to $\operatorname{crit}\left(F_{\epsilon}\right)$. As before it is enough to define $\pi_{\epsilon+1}$ on $\mathfrak{D}\left(\mathcal{P}_{\epsilon+1}\right)$, but now $\mathfrak{D}\left(\mathcal{P}_{\epsilon+1}\right)=\operatorname{Ult}_{0}\left(\mathfrak{D}(\overline{\mathcal{P}}), F_{\epsilon}\right)$. Let $\bar{\sigma}$ be the resurrection map $\sigma_{\eta_{\xi}, l_{\xi}}^{P_{\xi}^{*}}\left[\pi_{\xi}(\overline{\mathcal{P}})\right]$. For $f \in \mathfrak{D}(\overline{\mathcal{P}})$ and finite $a \subseteq \lambda_{\epsilon}$ set $\pi_{\epsilon+1}\left(i_{\xi, \epsilon+1}(f)(a)\right)=\left(i_{\xi, \epsilon+1}^{*} \circ \bar{\sigma} \circ \pi_{\xi}(f)\right)\left(h \circ \sigma_{\epsilon} \circ \pi_{\epsilon}(a)\right)$. We leave it to the reader to verify that this is well defined and preserves the conditions of Definition 2.2. The calculations for this are similar to the ones in the case of no truncation, but this time $\sigma_{\xi}$ is not the identify on $X \subseteq \pi_{\xi}\left(\operatorname{crit}\left(F_{\epsilon}\right)\right)$; instead, $\sigma_{\xi}(X)=\bar{\sigma}(X)$. This fact follows from the agreement between resurrections, since no strict initial segment of $\overline{\mathcal{P}}$ at or above $\mathcal{P}_{\xi} \|\left\langle\alpha_{\xi}, 0\right\rangle$ projects to $\operatorname{crit}\left(F_{\epsilon}\right)$. It affects line (7) of the computation. It also affects line $(3)$ if $\xi=\epsilon$, since $\sigma_{\epsilon}$ then agrees with $\bar{\sigma}$ rather than $i d$, on subsets of $\pi_{\epsilon}\left(\operatorname{crit}\left(F_{\epsilon}\right)\right)$.

The structure of the proof of Claim 2.3 is standard for fully backgrounded constructions. The only modification we made to the standard proof is in the addition of the function $h$, to take account of the fact that our certificates for $F$ do not extend $F$, but rather subsumes a stretch of $F$ by an embedding $h$.

LEMMA 2.4. Let $\delta$ be an inaccessible cardinal. Let $\mathcal{M}_{\nu, k}$ for $\langle\nu, k\rangle \leq_{\text {Lex }}\langle\delta, 0\rangle$ be given by the construction in Section 2 of Andretta-Neeman-Steel [1] but replacing the notion of certifiability with the one in our Definition 2.1.

Assume $\mathrm{SBH}_{\delta}$. Then the construction does not end at any $\langle\nu, k\rangle<_{\text {Lex }}\langle\delta, 0\rangle$, the model $\mathcal{W}=\mathcal{M}_{\delta, 0}$ has ordinal height $\delta$, and all countable elementary substructures of levels of $\mathcal{W}$ are $\omega_{1}+1$ iterable for normal, maximal, non-overlapping trees and their countable compositions. Moreover, $\mathcal{W}$ satisfies the following maximality condition:

Suppose $\kappa<\lambda<\delta$ are cardinals of $\mathcal{W}, \alpha \leq\left(\lambda^{+}\right)^{\mathcal{W}}$, and $F: \mathcal{W}\left\lceil\left(\kappa^{+}\right)^{\mathcal{W}} \rightarrow \mathcal{W}\lceil\alpha\right.$ (cofinal) is an extender over $\mathcal{W}$ with $\operatorname{crit}(F)=\kappa$ and $F(\operatorname{crit}(F))=\lambda$. Suppose that in $V_{\delta}$ there is an extender $F^{*}$ over $V$ and an embedding $h$ so that $\operatorname{crit}\left(F^{*}\right)=\kappa, F^{*}$ is $\eta$ strong for some inaccessible cardinal $\eta>\lambda$, $\operatorname{crit}(h) \geq \lambda$, and $h \circ F \subseteq i_{F^{*}}$ where $i_{F^{*}}$ is the ultrapower embedding of $V$ by $F^{*}$. Then $F$ is on the sequence of $\mathcal{W}$, indexed at $\alpha$.

ProOf. We already discussed the fact that the conversion of trees on $\mathcal{M}_{\nu, k}$ whose successor case (the only non-trivial case) is given by Claim 2.3 allows reducing iteration games on countable substructures of $\mathcal{W}$ and its initial segments to coarse iteration games on transitive collapses of countable $H \prec V_{\delta}$, which in 
turn the good player wins by $\mathrm{SBH}_{\delta}$. The facts that the construction of $\mathcal{M}_{\nu, k}$ proceeds to $\langle\delta, 0\rangle$, that $\mathcal{M}_{\delta, 0}$ has ordinal height $\delta$, and that $\mathcal{M}_{\delta, 0}$ is iterable in the manner described in the lemma, all follow from this by Theorem 2.21 of Andretta-Neeman-Steel [1].

Theorem 2.21 of Andretta-Neeman-Steel [1] also gives that no level $\mathcal{M}_{\nu, 0}$ is a bicephalus. This means that for every $\nu<\delta$, there can be at most one extender $F$ over $\mathcal{M}_{\nu, 0}^{-}$which is certifiable in the sense of Definition 2.1. By the construction, if there is such an extender $F$, then the top extender of $\mathcal{M}_{\nu, 0}$ is set to be equal to this $F$. The maximality condition in the lemma follows from this, taking $\langle\nu, 0\rangle=\operatorname{Res}_{\delta, 0}[\mathcal{W} \|\langle\alpha, 0\rangle]$, so that (by agreement properties of the resurrections, and since no level of $\mathcal{W}$ projects across $\lambda), \mathcal{M}_{\nu, 0}=\mathcal{W} \|\langle\alpha, 0\rangle$.

REMARK 2.5. If $\alpha$ indexes an extender in $\mathcal{W}$, then $\alpha$ is not a cardinal of $\mathcal{W}$. From this and the maximality condition in Lemma 2.4 it follows that if $\alpha \leq\left(\lambda^{+}\right)^{\mathcal{W}}$ and the assumptions of the maximality condition hold at $\alpha$, then in fact $\alpha<\left(\lambda^{+}\right)^{\mathcal{W}}$.

REMARK 2.6. The only uses of coarse iterability in the proof of Lemma 2.4 are with iteration trees on collapses of countable $H \prec V_{\delta}$ that arise through conversions from fine structural trees that are normal, maximal, and non-overlapping. The converted trees have several properties (for example on closure of strengths of extenders used), and the lemma goes through if $\mathrm{SBH}_{\delta}$ is restricted to trees that have these properties. Further, any assumption that produces cSBH of NeemanSteel [8] (under Jensen indexing and with the background condition modified to our current one) is enough, including in particular the assumption cUBH of [8] for our context.

§3. Stacking. Call a premouse $\mathcal{M}$ weakly iterable if the good player wins the length $\omega_{1}+1$ iteration game for normal, maximal, non-overlapping trees and their linear compositions, on all countable elementary substructures of $\mathcal{M}$. One of the tools we use to create fine structural models is stacking weakly iterable premice. This section includes some results on stacking that we will need for this purpose. With the exception of Lemma 3.2 and Claim 3.11, all the results in this section are either the obvious generalizations (with the same proofs) of Jensen's results in [2] for stacking over general premice rather than initial segments of $K^{c}$ and for allowing superstrong extenders, or slight adaptations and immediate consequences of such generalizations. Lemma 3.2 and Claim 3.11 are new though the methods used to prove them are similar.

Lemma 3.1. Let $\mathcal{M}_{i}$, for $i=1,2$, be sound weakly iterable premice extending a common initial segment $\mathcal{N}$, with $\rho\left(\mathcal{M}_{1}\right)=\rho\left(\mathcal{M}_{2}\right)=\operatorname{Ord} \cap \mathcal{N}$. Suppose further that $\operatorname{Ord} \cap \mathcal{N}$ is a regular uncountable cardinal. Then $\mathcal{M}_{1}$ and $\mathcal{M}_{2}$ are comparable, meaning that one is an initial segment of (or equal to) the other.

Proof. This is a direct generalization of Lemma 3.1 of [2]. Let $\lambda=\operatorname{Ord} \cap \mathcal{N}$. For $\nu<\lambda$, let $H_{i}^{\nu}$ be the elementary hull of $\nu \cup p\left(\mathcal{M}_{i}\right)$ in $\mathcal{M}_{i}$, let $\overline{\mathcal{M}}_{i}^{\nu}$ be the transitive collapse of $H_{i}^{\nu}$, and let $\sigma_{i}^{\nu}$ be the anticollapse embedding. (By elementary hull we mean the $r \Sigma_{k\left(\mathcal{M}_{i}\right)+1}$ hull. Equivalently, $\overline{\mathcal{M}}_{i}^{\nu}$ is the unique premouse so that $\mathfrak{D}\left(\overline{\mathcal{M}}_{i}^{\nu}\right)$ is the transitive collapse of the $\Sigma_{1}$ hull of $\nu \cup p\left(\mathcal{M}_{i}\right)$ 
in $\mathfrak{D}\left(\mathcal{M}_{i}\right)$.) Let $C_{i}$ be the set of $\nu$ so that $H_{i}^{\nu} \cap \lambda=\nu$, all bounded subsets of $\nu$ which are definable over $\mathcal{M}_{i}$ (by the level of elementarity above) from parameters in $H_{i}^{\nu}$ belong to $H_{i}^{\nu}$, and the theory of $\mathcal{M}_{i}$ (again restricted to the same level of elementarity) differs from each set in $H_{i}^{\nu}$ on some statement with parameters in $\nu \cup p\left(\mathcal{M}_{i}\right)$. Then $C_{i}$ is club in $\lambda$, and for each $\nu \in C_{i}$, $\operatorname{crit}\left(\sigma_{i}^{\nu}\right)=\nu$ and $\rho\left(\overline{\mathcal{M}}_{i}^{\nu}\right)=\nu$.

By condensation, $\overline{\mathcal{M}}_{i}^{\nu}$ is a proper initial segment of $\mathcal{N}$ if $E_{\nu}^{\mathcal{N}}=\emptyset$, and of $\operatorname{Ult}\left(\mathcal{N}, E_{\nu}^{N}\right)$ if $E_{\nu}^{\mathcal{N}} \neq \emptyset$. (See Theorem 9.3.2 of Zeman [19] for the precise phrasing of the condensation lemma. Case (c) of the condensation lemma is avoided here because $\rho\left(\overline{\mathcal{M}}_{i}^{\nu}\right)=\nu . \mathcal{N}$ or its ultrapower suffices, with no need to go to $\mathcal{M}$ or its ultrapower, since $\left|\overline{\mathcal{M}}_{i}^{\nu}\right|<\lambda$. Since $\lambda$ is a cardinal, it does not index an extender in $\mathcal{M}_{i}$. Hence $\nu$ does not index an extender in $\overline{\mathcal{M}}_{i}^{\nu}$ and this rules out the possibility that $\overline{\mathcal{M}}_{i}^{\nu}$ is an initial segment of $\mathcal{N}$ in case $E_{\nu}^{\mathcal{N}} \neq \emptyset$.)

It follows from the above that for any $\nu \in C_{1} \cap C_{2}, \overline{\mathcal{M}}_{1}$ and $\overline{\mathcal{M}}_{2}$ are either both strict initial segments of $\mathcal{N}$, or both strict initial segments of $\operatorname{Ult}\left(\mathcal{N}, E_{\nu}\right)$. Hence in particular they are comparable.

Since $\lambda$ is regular and uncountable, we can find $\nu \in C_{1} \cap C_{2}$ and $H \prec V_{\theta}$, for any large enough $\theta$, with $\lambda, \mathcal{M}_{1}, \mathcal{M}_{2} \in H$ and $H \cap \lambda=\nu$, so that $H_{i}^{\nu}$ is exactly equal to $H \cap \mathcal{M}_{i}$. Then $\overline{\mathcal{M}}_{i}^{\nu}$ is exactly equal to $c\left(\mathcal{M}_{i}\right)$ where $c$ is the collapse embedding of $H$. Since $c^{-1}$ is elementary, the comparability of $\overline{\mathcal{M}}_{1}^{\nu}$ and $\overline{\mathcal{M}}_{2}^{\nu}$ now implies the comparability of $\mathcal{M}_{1}$ and $\mathcal{M}_{2}$.

We will need the following strengthening of Lemma 3.1, which allows for some situations where the height of $\mathcal{N}$ is not a cardinal.

Lemma 3.2. Let $\mathcal{M}_{i}$, for $i=1,2$, be sound weakly iterable premice extending a common initial segment $\mathcal{N}$, with $\rho\left(\mathcal{M}_{i}\right)=\operatorname{Ord} \cap \mathcal{N}$. Suppose $\operatorname{Cof}(\operatorname{Ord} \cap$ $\mathcal{N})$ is uncountable. Suppose further for each $i$, $\operatorname{Ord} \cap \mathcal{N}$ is regular relative to functions elementarily definable with parameters over $\mathcal{M}_{i}$. Then $\mathcal{M}_{1}$ and $\mathcal{M}_{2}$ are comparable.

Elementary definability in the lemma (and throughout the section) is fine structural, meaning $r \Sigma_{k\left(\mathcal{M}_{i}\right)+1}$ over $\mathcal{M}_{i}$, as in Section 2 .

Proof of Lemma 3.2. Let $\gamma=\operatorname{Ord} \cap \mathcal{N}$. For $\nu<\gamma$, let $H_{i}^{\nu}$ be the elementary hull of $\nu \cup p\left(\mathcal{M}_{i}\right)$ in $\mathcal{M}_{i}$, let $\overline{\mathcal{M}}_{i}^{\nu}$ be the transitive collapse of $H_{i}^{\nu}$, and let $\sigma_{i}^{\nu}$ be the anticollapse embedding. Note $\overline{\mathcal{M}}_{i}^{\nu} \in \mathcal{M}_{i}$ since $\rho\left(\mathcal{M}_{i}\right)>\nu$. Let $C_{i}$ be a club in $\gamma$ so that for every $\nu \in C_{i}, H_{i}^{\nu} \cap \gamma=\nu$. Such a club exists since $H_{i}^{\nu}$ is always bounded in $\gamma$, a consequence of the fact that $\gamma$ is regular relative to functions that are elementarily definable over $\mathcal{M}_{i}$, and since $\operatorname{Cof}(\gamma)$ is uncountable. Thinning $C_{i}$ if needed we can also make sure for $\nu \in C_{i}$ that there are cofinally many $\mu<\nu$ so that $\overline{\mathcal{M}}_{i}^{\mu} \in \overline{\mathcal{M}}_{i}^{\nu}$. This implies that $\rho\left(\overline{\mathcal{M}}_{i}^{\nu}\right)=\nu$.

By condensation, for each $\nu \in C_{i}, \overline{\mathcal{M}}_{i}^{\nu}$ is an initial segment of $\mathcal{N}$ if $E_{\nu}^{\mathcal{N}}=\emptyset$, and of $\operatorname{Ult}\left(\mathcal{N}, E_{\nu}^{\mathcal{N}}\right)$ if $E_{\nu}^{\mathcal{N}} \neq \emptyset$. (Case (c) of the condensation lemma is ruled out because $\rho\left(\mathcal{M}_{i}^{\nu}\right)=\nu$. Since $\gamma$ is a cardinal in $\mathcal{M}_{i}$ it does not index an extender. Hence $\nu$ does not index an extender in $\overline{\mathcal{M}}_{i}$, so the remaining cases of condensation can be separated, to conclude that $\overline{\mathcal{M}}_{i}$ is an initial segment of $\mathcal{M}_{i}$ if $E_{\nu}^{\mathcal{M}_{i}}=E_{\nu}^{\mathcal{N}}=\emptyset$, and of $\operatorname{Ult}\left(\mathcal{M}_{i}, E_{\nu}^{\mathcal{N}}\right)$ otherwise. $\mathcal{N}$ and the ultrapower of $\mathcal{N}$ suffice to subsume $\overline{\mathcal{M}}_{i}^{\nu}$ in the two cases, since $\overline{\mathcal{M}}_{i}^{\nu}$ has cardinality $\nu<\gamma$ in $\mathcal{M}_{i}$.) 
For $i=1,2$ let $S_{i}=\left\{\xi \in C \mid \overline{\mathcal{M}}_{i}^{\xi} \triangleleft \overline{\mathcal{M}}_{3-i}^{\xi}\right\}$. Let $S_{3}=\left\{\xi \in C \mid \overline{\mathcal{M}}_{1}^{\xi}=\overline{\mathcal{M}}_{2}^{\xi}\right\}$. Then by the above, $S_{1} \cup S_{2} \cup S_{3} \supseteq C_{1} \cap C_{2}$. So at least one of $S_{1}, S_{2}, S_{3}$ is stationary in $\gamma$.

For $\xi<\zeta$ both in $C_{1} \cap C_{2}$ let $\sigma_{i}^{\xi, \zeta}$ be the natural embedding of $\overline{\mathcal{M}}_{i}^{\xi}$ into $\overline{\mathcal{M}}_{i}^{\zeta}$ induced by the identify on $H_{i}^{\xi}$. Equivalently this is the unique embedding so that $\sigma_{i}^{\xi}=\sigma_{i}^{\zeta} \circ \sigma_{i}^{\xi, \zeta}$.

Note that $\mathcal{M}_{i}$ is the direct limit of the system $\mathcal{D}_{i}^{S}=\left\langle\overline{\mathcal{M}}_{i}^{\xi}, \sigma_{i}^{\xi, \zeta} \mid \xi<\zeta \in S\right\rangle$, for any $S \subseteq C_{1} \cap C_{2}$ cofinal, and that $\sigma_{i}^{\xi}$ are the direct limit embeddings. Note further that the map $\sigma_{i}^{\xi, \zeta}$ is determined from $\overline{\mathcal{M}}_{i}^{\zeta}$ and from $\xi$, independently of $i$ : It is the anticollapse embedding of the elementary hull of $\xi \cup p\left(\overline{\mathcal{M}}_{i}^{\zeta}\right)$ in $\overline{\mathcal{M}}_{i}^{\zeta}$.

If $S_{3}$ is stationary, then it follows from the previous paragraph that $\mathcal{M}_{1}$ and $\mathcal{M}_{2}$ are equal, since the systems $\mathcal{D}_{i}^{S_{3} \cap C_{1} \cap C_{2}}$ for $i=1,2$ are the same.

Suppose $S_{1}$ is stationary. Note that for every limit $\zeta \in S_{1}$, and all sufficiently large $\xi<\zeta$ in $C_{1} \cap C_{2}$, the elementary hull of $\xi \cup p\left(\overline{\mathcal{M}}_{1}^{\zeta}\right)$ in $\overline{\mathcal{M}}_{1}^{\zeta}$ is exactly equal to the intersection of $\overline{\mathcal{M}}_{1}^{\zeta}$ with the elementary hull of $\xi \cup p\left(\overline{\mathcal{M}}_{2}^{\zeta}\right)$ in $\overline{\mathcal{M}}_{2}^{\zeta}$. This can be seen using the elementarity of the latter hull, and the fact that $\overline{\mathcal{M}}_{1}^{\zeta} \triangleleft \overline{\mathcal{M}}_{2}^{\zeta}$.

It follows that for limit $\zeta \in S_{1}$ and all sufficiently large $\xi<\zeta$ in $C_{1} \cap C_{2}$, $\overline{\mathcal{M}}_{1}^{\zeta}=\sigma_{2}^{\xi, \zeta}\left(\overline{\mathcal{M}}_{1}^{\xi}\right)$ and $\sigma_{1}^{\xi, \zeta}=\sigma_{2}^{\xi, \zeta} \uparrow \overline{\mathcal{M}}_{1}^{\xi}$. Since $\operatorname{Cof}(\gamma)$ is uncountable and $S_{1}$ is stationary, we can find $\alpha<\gamma$ in $S_{1} \cap C_{1} \cap C_{2}$ so that "all sufficiently large $\xi$ " above can be replaced by "all $\xi \geq \alpha$ ". Then the direct limit of $\mathcal{D}_{1}^{S_{1} \cap C_{1} \cap C_{2}}$ is exactly equal to $\sigma_{2}^{\alpha}\left(\overline{\mathcal{M}}_{1}^{\alpha}\right)$. Since this direct limit is $\mathcal{M}_{1}$ it follows that $\mathcal{M}_{1} \triangleleft \mathcal{M}_{2}$.

A similar argument shows that $\mathcal{M}_{2} \triangleleft \mathcal{M}_{1}$ in the case that $S_{2}$ is stationary. $\dashv$

Definition 3.3. Let $\lambda$ be an uncountable regular cardinal.

1. For $\mathcal{N}$ a premouse of ordinal height $\lambda$, let $U(\mathcal{N})$ be the set of all sound weakly iterable premice $\mathcal{M}$ extending $\mathcal{N}$ with $\rho(\mathcal{M})=\lambda$.

2. For $\mathcal{N}$ a premouse of ordinal height $\gamma>\lambda$ with $\operatorname{Cof}(\gamma)=\lambda$, let $U(\mathcal{N})$ be the set of all sound weakly iterable premice $\mathcal{M}$ extending $\mathcal{N}$ so that $\rho(\mathcal{M})=\gamma$ and $\gamma$ is regular relative to functions elementarily definable with parameters over $\mathcal{M}$.

By Lemmas 3.1 and 3.2, the premice in $U(\mathcal{N})$ are comparable. Hence they can be put together to form a premouse extending $\mathcal{N}$. Precisely:

Definition 3.4. Let $\mathcal{S}(\mathcal{N})$ be the unique premouse so that:

1. All elements of $U(\mathcal{N})$ are strict initial segments of $\mathcal{S}(\mathcal{N})$.

2. Every strict initial segment of $\mathcal{S}(\mathcal{N})$ is or extends to an element of $U(\mathcal{N})$.

3. If $k(\mathcal{S}(\mathcal{N}))=0$ then $\mathcal{S}(\mathcal{N})$ is passive.

We mostly use $\mathcal{S}(\mathcal{N})$ in contexts where $\mathcal{N}$ is weakly iterable, $\rho(\mathcal{N})=\mathcal{N} \cap$ Ord, and $\mathcal{N} \cap$ Ord is regular relative to functions elementarily definable with parameters over $\mathcal{N}$. Then $\mathcal{N}$ itself belongs to $U(\mathcal{N})$, and hence $\mathcal{S}(\mathcal{N})$ extends $\mathcal{N}$ strictly.

If $U(\mathcal{N})$ has a maximal element $\mathcal{M}$, then $\mathcal{S}(\mathcal{N})$ is the premouse with the same sequence and ordinal height as $\mathcal{M}$, and with $k(\mathcal{S}(\mathcal{N}))=k(\mathcal{M})+1$. In this case $\mathcal{S}(\mathcal{N})$ may be active with the same top predicate as $\mathcal{M}$. If $U(\mathcal{N})$ does not have a maximal element, then $\mathcal{S}(\mathcal{N})$ is the least premouse extending all elements of $U(\mathcal{N})$. In this case $k(\mathcal{S}(\mathcal{N}))=0$ and $\mathcal{S}(\mathcal{N})$ is passive. 
Corollary 3.5. Let $\lambda$ be a regular uncountable cardinal. Let $\mathbb{P}$ be a poset. Suppose that forcing with $\mathbb{P} \times \mathbb{P}$ does not change the cofinality of $\lambda$ to countable. Let $\mathcal{N}$ be a premouse with ordinal height of cofinality $\lambda$ in $V$ (possibly ordinal height equal to $\lambda$ ). Then all elements of $U(\mathcal{N})^{V^{\mathbb{P}}}$ belong to $V$. If $\mathbb{P}$ does not add sets of reals, so that premice in $V$ are weakly iterable in $V$ iff they are weakly iterable in $V^{\mathbb{P}}$, then $U(\mathcal{N})^{V^{\mathbb{P}}}=U(\mathcal{N})^{V}$.

Proof. We prove the initial part of the corollary; the final sentence of the corollary is then an immediate consequence. Fix a name $\dot{\mathcal{M}} \in V$ for a premouse in $U(\mathcal{N})^{V^{\mathbb{P}}}$. Suppose for contradiction that $p \in \mathbb{P}$ forces the interpretation of $\dot{\mathcal{M}}$ to not belong to $V$. Let $G_{1} \times G_{2}$ be generic for $\mathbb{P} \times \mathbb{P}$ with $\langle p, p\rangle \in G_{1} \times G_{2}$. Let $\mathcal{M}_{i}=\dot{\mathcal{M}}\left[G_{i}\right]$. By Lemma 3.2 the premice $\mathcal{M}_{1}$ and $\mathcal{M}_{2}$ are comparable. (The lemma is used in $V\left[G_{1} \times G_{2}\right]$, where $\operatorname{Cof}(\lambda)$ remains uncountable. As stated a use of the lemma in $V\left[G_{1} \times G_{2}\right]$ requires weak iterability of $\mathcal{M}_{i}$ in $V\left[G_{1} \times G_{2}\right]$. But the only use of weak iterability in the proof of the lemma is for the application of the condensation lemma. Condensation is used separately on each $\mathcal{M}_{i}$. So the weak iterability of $\mathcal{M}_{i}$ in $V\left[G_{i}\right]$ is sufficient.) Let $i$ be such that $\mathcal{M}_{i} \unlhd \mathcal{M}_{3-i}$. Then $\mathcal{M}_{i}=\dot{\mathcal{M}}\left[G_{i}\right]$ belongs to both $V\left[G_{i}\right]$ and $V\left[G_{3-i}\right]$, and this implies that it belongs to $V$.

LEMMA 3.6. Let $\lambda$ be a regular uncountable cardinal and let $\mathcal{N}$ be a premouse of ordinal height $\lambda$. Suppose $|\mathcal{S}(\mathcal{N})|=\lambda$ and let $\left\langle H_{\xi} \mid \xi<\lambda\right\rangle$ be a continuous increasing chain with $\left|H_{\xi}\right|<\lambda$ and $\bigcup_{\xi<\lambda} H_{\xi}=\mathcal{S}(\mathcal{N})$. Then there is a club $C \subseteq \lambda$ so that for every $\xi \in C$ :

1. $H_{\xi} \cap \lambda=\xi$.

2. Every strict initial segment of the transitive collapse of $H_{\xi}$ is an initial segment of $\mathcal{N}$, below the successor of $\xi$ in $\mathcal{N}$.

Proof. Fix $\left\{\mathcal{M}_{\alpha} \in U(\mathcal{N}) \mid \alpha<\beta\right\}$ cofinal in the strict initial segments of $\mathcal{S}(\mathcal{N})$, with $\beta \leq \lambda$. This is possible by definition of $\mathcal{S}(\mathcal{N})$ and the assumption that $|\mathcal{S}(\mathcal{N})|=\lambda$. For each $\xi<\lambda$, let $H_{\xi}^{\alpha}$ be the elementary hull of $\xi \cup p\left(\mathcal{M}_{\alpha}\right)$ in $\mathcal{M}_{\alpha}$. By condensation, or the proof of Lemma 3.1, there is a club $C^{\alpha} \subseteq \lambda$ of $\xi$ so that the transitive collapse of $H_{\xi}^{\alpha}$ is an initial segment of $\mathcal{N}$, below the successor of $\xi$ in $\mathcal{N}$ since the transitive collapse projects to $\xi$.

Abusing notation slightly, we work below as if all $\mathcal{M}_{\alpha}$ belong to $\mathcal{S}(\mathcal{N})$. This is an abuse of notation, since $\mathcal{S}(\mathcal{N})$ may be a $k$-premouse for some $k \geq 1$, in which case its obvious restriction to a $j$-premouse for some $j<k$ (in fact cofinally many $j<k$ ) belongs to $\left\{\mathcal{M}_{\alpha} \in U(\mathcal{N}) \mid \alpha<\beta\right\}$. Any $\mathcal{M}_{\alpha}$ which is equal to such a restriction does not belong to $\mathcal{S}(\mathcal{N})$. But it is coded into the master structure of $\mathcal{S}(\mathcal{N})$, so there is enough elementarity for maps to and from $\mathcal{S}(\mathcal{N})$ to still carry out arguments below that are phrased as if $\mathcal{M}_{\alpha} \in \mathcal{S}(\mathcal{N})$.

Suppose $\left\langle H_{\xi} \mid \xi<\lambda\right\rangle$ is a continuous increasing chain with $\left|H_{\xi}\right|<\lambda$ and $\bigcup_{\xi<\lambda} H_{\xi}=\mathcal{S}(\mathcal{N})$. By standard arguments using the regularity of $\lambda$, there is a club $C$ of $\xi<\lambda$ for which $H_{\xi} \cap \lambda=\xi, H_{\xi}$ is elementary in $\mathcal{S}(\mathcal{N}),\left\{\mathcal{M}_{\alpha} \mid \alpha<\right.$ $\beta\} \cap H_{\xi}$ is cofinal in the strict initial segments of $\mathcal{S}(\mathcal{N})$ which belong to $H_{\xi}$, and $\mathcal{M}_{\alpha} \in H_{\xi} \rightarrow \xi \in C^{\alpha}$. Fix $\xi \in C$ and let $c$ be the transitive collapse embedding on $H_{\xi}$. Then $\left\{c\left(\mathcal{M}_{\alpha}\right) \mid \mathcal{M}_{\alpha} \in H_{\xi}\right\}$ is cofinal in the strict initial segments of the transitive collapse of $H_{\xi}$. Fix $\alpha$ with $\mathcal{M}_{\alpha} \in H_{\xi}$. The elementarity of $H_{\xi}$ implies 
that $H_{\xi} \cap \mathcal{M}_{\alpha}=H_{\xi}^{\alpha}$. It follows that $c\left(\mathcal{M}_{\alpha}\right)$ is exactly the transitive collapse of $H_{\xi}^{\alpha}$. Since $\xi \in C^{\alpha}$ this implies that $c\left(\mathcal{M}_{\alpha}\right)$ is an initial segment of $\mathcal{N}$ below the successor of $\xi$ in $\mathcal{N}$.

Assuming $\mathcal{N}$ itself is weakly iterable, so that $U(\mathcal{N}) \neq \emptyset$ and $\mathcal{S}(\mathcal{N})$ extends $\mathcal{N}$, the assumption in Lemma 3.6 that $|\mathcal{S}(\mathcal{N})|=\lambda$ can always be secured by collapsing the ordinal height of $\mathcal{S}(\mathcal{N})$ to $\lambda$. The collapse does not change $\mathcal{S}(\mathcal{N})$, by Corollary 3.5.

For later applications we will want to ensure that, using the terminology of Lemma 3.6, for a large set of $\xi$, the transitive collapse of $H_{\xi}$ is exactly equal to $\mathcal{N} \uparrow\left(\xi^{+}\right)^{\mathcal{N}}$. We will have our own method for securing this, under additional assumptions, in case the cofinality of $\operatorname{Ord} \cap \mathcal{S}(\mathcal{N})$ is $\lambda$. The case of smaller cofinality can be managed more generally. This is done in Lemma 3.9. The proof is essentially the main part of the proof of Theorem 3.4 of [2].

Claim 3.7. Let $\lambda$ be a regular uncountable cardinal. Let $\mathcal{N}$ be a premouse of ordinal height $\lambda$. Suppose $\lambda$ is a limit of cardinals of $\mathcal{N}$, and $\mathcal{N}$ is weakly iterable. Then there is no premouse $\mathcal{P} \unrhd \mathcal{S}(\mathcal{N})$ so that all extenders on the sequence of $\mathcal{P}$ are on the sequence of $\mathcal{S}(\mathcal{N})$ (where we consider top extenders, if they exist, to be on the sequence) and $\rho(\mathcal{P}) \leq \lambda$.

Proof. Similar to the proof of Lemma 3.3 of [2]. Suppose the claim fails, and let $\mathcal{P}$ witness this. Fix some large regular $\theta$, and let $H \prec V_{\theta}$ with $H \cap \lambda=\tau$ for an ordinal $\tau<\lambda,|H|<\lambda$, and $\mathcal{P}, \mathcal{S}(\mathcal{N}), \lambda \in H$. Since $\lambda$ is a limit of cardinals in $\mathcal{N}$ we may pick $H$ so that $\tau$ is a cardinal of $\mathcal{N}$. Let $c$ be the transitive collapse map of $H$.

By elementarity, $c(\mathcal{P})$ has no extenders on its sequence other than ones on the sequence of $c(\mathcal{S}(\mathcal{N}))$. If $c(\mathcal{S}(\mathcal{N}))$ has a top extender then by definition of $\mathcal{S}(\mathcal{N})$ it must be that $k(c(\mathcal{S}(\mathcal{N})))=k(\mathcal{S}(\mathcal{N}))>0$, hence the top extender is part of a strict initial segment of $c(\mathcal{S}(\mathcal{N}))$. Any earlier extenders are also on strict initial segments of $c(\mathcal{S}(\mathcal{N}))$. By Lemma 3.6, every strict initial segment of $c(\mathcal{S}(\mathcal{N}))$ is an initial segment of $\mathcal{N}$. Combining these facts, plus the fact that $c(\mathcal{P})$ extends $c(\mathcal{S}(\mathcal{N}))$ and has ordinal height below $\lambda$, it follows that the active part of the extender sequence of $c(\mathcal{P})$ is an initial segment of the active part of the extender sequence of $\mathcal{N}$, and that $c(\mathcal{P}) \subseteq \mathcal{N}$.

In particular $c(\mathcal{P})$ is weakly iterable. Further, $\rho(c(\mathcal{P})) \geq \tau$, since $\tau$ is a cardinal of $\mathcal{N}$. Both these facts reflect from $V$ to the transitive collapse of $H$, so using the elementarity of $c^{-1}, \mathcal{P}$ is weakly iterable and $\rho(\mathcal{P}) \geq \lambda$. Since $\rho(\mathcal{P}) \leq \lambda$ by assumption, it must be that $\rho(\mathcal{P})=\lambda$. Replacing $\mathcal{P}$ by its core, which still extends $\mathcal{S}(\mathcal{N})$ by standard universality arguments and projects to $\lambda$, we may assume it is sound. But then $\mathcal{P} \in U(\mathcal{N})$, contradicting the fact that $\mathcal{P} \unrhd \mathcal{S}(\mathcal{N})$

REMARK 3.8. The conclusion of Claim 3.7 implies that $k(\mathcal{S}(\mathcal{N}))=0$, and in particular every strict initial segment of $\mathcal{S}(\mathcal{N})$ is an element of $\mathcal{S}(\mathcal{N})$. For otherwise $\mathcal{S}(\mathcal{N})$ has a largest strict initial segment, by definition of $\mathcal{S}(\mathcal{N})$ this initial segment must project to $\lambda$, and this implies that $\mathcal{S}(\mathcal{N})$ itself projects to or below $\lambda$, contradicting Claim 3.7. By condition (3) of Definition 3.4 it also follows from Claim 3.7 that $\mathcal{S}(\mathcal{N})$ is passive. With this additional information one can 
strengthen the conclusion of Lemma 3.6. To be precise, under the conditions of the lemma, and the additional assumptions in Claim 3.7, for a club of $\xi<\lambda$ (in fact for all $\xi$ in the club defined during the proof of Lemma 3.6), $H_{\xi} \cap \lambda=\xi$, $H_{\xi}$ is elementary in $\mathcal{S}(\mathcal{N})$, and the transitive collapse of $H_{\xi}$ is equal to $\mathcal{N} \uparrow \mu$ for some $\mu \leq\left(\xi^{+}\right)^{\mathcal{N}}$.

Lemma 3.9. Let $\lambda$ be a regular uncountable cardinal. Suppose that $\tau<\lambda \rightarrow$ $\tau^{\omega}<\lambda$. Let $\mathcal{N}$ be a weakly iterable premouse of ordinal height $\lambda$, and suppose $\lambda$ is a limit of cardinals of $\mathcal{N}$. Let $\eta=\operatorname{Cof}(\mathcal{S}(\mathcal{N}) \cap$ Ord $)$, and suppose $\eta<\lambda$. Let $\left\langle H_{\xi} \mid \xi<\lambda\right\rangle$ be a continuous increasing chain with $\left|H_{\xi}\right|<\lambda$ and $\bigcup_{\xi<\lambda} H_{\xi}=$ $\mathcal{S}(\mathcal{N})$. Then there is a club of $\xi<\lambda$ so that, except possibly if $\operatorname{Cof}(\xi) \in\left\{\omega, \omega_{1}, \eta\right\}$, the transitive collapse of $H_{\xi}$ is equal to $\mathcal{N} \uparrow\left(\xi^{+}\right)^{\mathcal{N}}$.

Proof. Let $\mathcal{R}_{\xi}$ denote the transitive collapse of $H_{\xi}$, and let $\pi_{\xi}: \mathcal{R}_{\xi} \rightarrow \mathcal{S}(\mathcal{N})$ be the anticollapse embedding. Let $\pi_{\xi, \zeta}: \mathcal{R}_{\xi} \rightarrow \mathcal{R}_{\zeta}$ be the map $\pi_{\zeta}^{-1} \circ \pi_{\xi}$.

Let $S$ be the set of $\xi<\lambda$ so that $\mathcal{R}_{\xi} \neq \mathcal{N} \uparrow\left(\xi^{+}\right)^{\mathcal{N}}$. Suppose for contradiction that $\left\{\xi \in S \mid \operatorname{Cof}(\xi) \notin\left\{\omega, \omega_{1}, \eta\right\}\right\}$ is stationary.

Let $C$ be the club witnessing Lemma 3.6 and Remark 3.8, intersected with the set of cardinals of $\mathcal{N}$, which by the assumptions of the lemma is club in $\lambda$. Then for every $\xi \in S \cap C, \mathcal{R}_{\xi}$ is equal to a restriction of $\mathcal{N}$ to a level strictly below $\left(\xi^{+}\right)^{\mathcal{N}}$. Since $\xi$ is a cardinal of $\mathcal{N}$ it follows that there is a level of $\mathcal{N}$ at or above $\mathcal{R}_{\xi}$ which projects to $\xi$. Let $\mathcal{P}_{\xi}$ be the least such. By the elementarity of $\pi_{\xi}$ and since cofinally many strict initial segments of $\mathcal{S}(\mathcal{N})$ project to $\lambda, \xi$ is the largest cardinal in $\mathcal{R}_{\xi} \cdot \mathcal{P}_{\xi}$ is then the least level of $\mathcal{N}$ at or above $\mathcal{R}_{\xi}$ which projects anywhere strictly below $\operatorname{Ord} \cap \mathcal{R}_{\xi}$.

For $\xi \in S \cap C$, let $F_{\xi}$ be the $(\xi, \lambda)$ extender derived from $\pi_{\xi}$. More generally, for $\xi<\zeta$ both in $S \cap C$, let $F_{\xi, \zeta}$ be the $(\xi, \zeta)$ extender derived from $\pi_{\xi, \zeta \text {. }}$. By the minimality of $\mathcal{P}_{\xi}$, the ultrapower $\operatorname{Ult}\left(\mathcal{P}_{\xi}, F_{\xi}\right)$ makes sense. (Recall that this ultrapower is defined so that $\mathfrak{D}\left(\operatorname{Ult}\left(\mathcal{P}_{\xi}, F_{\xi}\right)\right)=\operatorname{Ult}_{0}\left(\mathfrak{D}\left(\mathcal{P}_{\xi}\right), F_{\xi}\right)$. Since $\mathcal{P}_{\xi}$ is the least level of $\mathcal{N}$ projecting across Ord $\cap \mathcal{R}_{\xi}, \mathfrak{D}\left(\mathcal{P}_{\xi}\right)$ contains $\mathcal{R}_{\xi}$. Since $\mathfrak{D}\left(\mathcal{P}_{\xi}\right) \subseteq \mathcal{P}_{\xi}$ it follows that the subsets of $\xi$ in $\mathfrak{D}\left(\mathcal{P}_{\xi}\right)$ are exactly the ones in $\mathcal{R}_{\xi}$. So $\operatorname{Ult}_{0}\left(\mathfrak{D}\left(\mathcal{P}_{\xi}\right), F_{\xi}\right)$ makes sense.) Thinning $C$ if needed we may assume $\xi$ is a limit cardinal of $\mathcal{N}$, so if $\mathcal{P}_{\xi}$ has a top extender, the cardinal successor of its critical point is not $\xi$. This is important since it implies the top extender of the ultrapower is total, a requirement for being a premouse.

Thinning $C$ if needed we may assume that for all $\xi \in C, \pi_{\xi}$ is cofinal in $\mathcal{S}(\mathcal{N})$; this makes use of our assumption that $\operatorname{Cof}(\mathcal{S}(\mathcal{N}) \cap$ Ord $)=\eta<\lambda$. It is then easy to check $\operatorname{Ult}_{0}\left(\mathfrak{D}\left(\mathcal{P}_{\xi}\right), F_{\xi}\right)$ contains $\mathcal{S}(\mathcal{N})$. It follows that so does $\operatorname{Ult}\left(\mathcal{P}_{\xi}, F_{\xi}\right)$.

It is enough now to find some $\xi \in S \cap C$ so that $\operatorname{Ult}\left(\mathcal{P}_{\xi}, F_{\xi}\right)$ is weakly iterable, sound, and projects to $\lambda$. Then $\operatorname{Ult}\left(\mathcal{P}_{\xi}, F_{\xi}\right)$ belongs to $U(\mathcal{N})$, so it is a strict initial segment of $\mathcal{S}(\mathcal{N})$, hence by Remark 3.8 it is an element of $\mathcal{S}(\mathcal{N})$, contradicting the conclusion of the previous paragraph.

That $\rho\left(\operatorname{Ult}\left(\mathcal{P}_{\xi}, F_{\xi}\right)\right) \leq \lambda$ is clear, since $\rho\left(\mathcal{P}_{\xi}\right)=\xi=\operatorname{crit}\left(F_{\xi}\right)$ and the support of $F_{\xi}$ is $\lambda$. It is clear for the same reason that $\operatorname{Ult}\left(\mathcal{P}_{\xi}, F_{\xi}\right)$ is $\lambda$-sound. Thinning $C$ using the assumption that $\tau<\lambda \rightarrow \tau^{\omega}<\lambda$, we can ensure that for any $\xi \in S \cap C$ of uncountable cofinality, any tree of height $\omega$ whose restrictions to finite heights belong to $H_{\xi}$, has a branch in $V$ iff it has a branch contained in 
$H_{\xi}$. Then for every such $\xi$, any countable substructure of $\operatorname{Ult}\left(\mathcal{P}_{\xi}, F_{\xi}\right)$ embeds into $\mathcal{P}_{\xi}$. So the weak iterability of $\mathcal{P}_{\xi}$ transfers to $\operatorname{Ult}\left(\mathcal{P}_{\xi}, F_{\xi}\right)$.

In light of the above, it is enough to find one $\xi \in S \cap C$ of uncountable cofinality, so that $\rho\left(\operatorname{Ult}\left(\mathcal{P}_{\xi}, F_{\xi}\right)\right) \nless \lambda$.

Since any bounded subset of $\lambda$ that is definable over $\operatorname{Ult}\left(\mathcal{P}_{\xi}, F_{\xi}\right)$ is definable (in exactly the same manner) over $\operatorname{Ult}\left(\mathcal{P}_{\xi}, F_{\xi, \zeta}\right)$ for any large enough $\zeta$, we can obtain $\rho\left(\operatorname{Ult}\left(\mathcal{P}_{\xi}, F_{\xi}\right)\right) \nless \lambda$ by ensuring that $\operatorname{Ult}\left(\mathcal{P}_{\xi}, F_{\xi, \zeta}\right) \in \mathcal{N}$ for arbitrarily large $\zeta<\lambda$.

To find $\xi$ we will use the following fine structural fact. The fact is phrased using the conventions of Andretta-Neeman-Steel [1], described in Section 2 above.

FACT 3.10. Let $\mathcal{P}$ be a premouse, $\rho=\rho(\mathcal{P}), p=p(\mathcal{P}), \mathfrak{D}=\mathfrak{D}(\mathcal{P})$ (so that $\rho$ and $p$ are the first projectum and standard parameter of $\mathfrak{D}$ ), and $\theta=\operatorname{Ord} \cap \mathfrak{D}$. Suppose $p$ is universal (a consequence, for example, of weak iterability for $\mathcal{P}$ ). Let $\delta=\left(\rho^{+}\right)^{\mathcal{P}}$, taking $\delta=\operatorname{Ord} \cap \mathcal{P}$ if $\rho$ is the largest cardinal of $\mathcal{P}$. Suppose no strict initial segment of $\mathcal{P}$ projects across $\delta$. Then:

1. $\delta$ and $\theta$ have the same cofinality in $V$.

2. If $\rho$ is regular in $\mathcal{P}$ but not regular relative to functions elementarily definable with parameters over $\mathcal{P}$, then $\rho$ and $\theta$ have the same cofinality in $V$, hence by condition $(1), \operatorname{Cof}(\rho)=\operatorname{Cof}(\delta)$.

Proof. Let $\nu$ be the cofinality of $\theta$ in $V$. Then $\mathfrak{D}$ can be written as an increasing union of structures $\mathfrak{D}_{i}, i<\nu$, which each belongs to $\mathfrak{D}$. Since no strict initial segment of $\mathcal{P}$ projects across $\delta, \theta \geq \delta$ and $\delta$ is not collapsed to $\rho$ in $\mathfrak{D}$. By the universality of the standard parameter $p$, the $\Sigma_{1}$ hull of $\rho \cup p$ in $\mathfrak{D}$ contains $\delta$. This hull is the increasing union of the hulls $H_{i}$ of $\rho \cup p$ in the structures $\mathfrak{D}_{i}$. For each $i<\nu, H_{i}$ can be computed inside $\mathfrak{D}$, and seen there to have cardinality at most $\rho$. Hence $\sup \left(H_{i}\right) \cap \delta<\delta$. The map $i \mapsto \sup \left(H_{i}\right) \cap \delta$ then witnesses that $\delta$ has cofinality $\nu$ in $V$.

Suppose that $\rho$ is regular in $\mathcal{P}$, but not regular relative to functions elementarily definable with parameters over $\mathcal{P}$. Elementarity here is in the fine structural sense over $\mathcal{P}$, meaning $\Sigma_{1}$ elementarity over $\mathfrak{D}$. Thus there is a finite $a \subseteq \mathfrak{D}$ and some $\xi<\rho$ so that the $\Sigma_{1}$ hull of $\xi \cup a$ in $\mathfrak{D}$ is cofinal in $\rho$. This hull is the increasing union of the hulls $H_{i}^{\prime}$ of $\xi \cup a$ in the structures $\mathfrak{D}_{i}$. Since $\rho$ is regular in $\mathcal{P}$, these hulls are each bounded in $\rho$. So the map $i \mapsto \sup \left(H_{i}^{\prime}\right) \cap \rho$ witnesses that $\rho$ has cofinality $\nu$ in $V$.

Returning now to the proof of Lemma 3.9, fix $\tau \in S \cap C$ so that $\tau$ is a limit point of $S \cap C, \operatorname{Cof}(\tau) \notin\left\{\omega, \omega_{1}, \eta\right\}$, and for every $\xi \in S \cap C \cap \tau$ of uncountable cofinality, either the set of $\zeta<\lambda$ so that $\operatorname{Ult}\left(\mathcal{P}_{\xi}, F_{\xi, \zeta}\right) \in \mathcal{N}$ is unbounded, or else its bound is smaller than $\tau$. Such $\tau$ can be obtained since $S$ is stationary outside the cofinalities $\omega, \omega_{1}$, and $\eta$. We will complete the proof by finding $\xi \in S \cap C \cap \tau$ of uncountable cofinality so that $\operatorname{Ult}\left(\mathcal{P}_{\xi}, F_{\xi, \tau}\right) \in \mathcal{N}$. By definition of $\tau$ this implies that there are unboundedly many $\zeta<\lambda$ with $\operatorname{Ult}\left(\mathcal{P}_{\xi}, F_{\xi, \zeta}\right) \in \mathcal{N}$, as required.

Since $\tau$ is the largest cardinal of $\mathcal{R}_{\tau}, k\left(\mathcal{R}_{\tau}\right)=0$, and $\operatorname{Cof}\left(\mathcal{R}_{\tau} \cap \operatorname{Ord}\right)=\eta$, there is a sequence $\left\langle\mathcal{M}_{\alpha} \mid \alpha<\eta\right\rangle$ of strict initial segments of $\mathcal{R}_{\tau}$, cofinal in $\mathcal{R}_{\tau}$, so that each $\mathcal{M}_{\alpha}$ projects to $\tau$ and belongs to $\mathcal{R}_{\tau}$. Since $\pi_{\tau}^{-1 " \prime} H_{\min (C)}$ is cofinal in $\mathcal{R}_{\tau}$, we can take $M_{\alpha} \in \pi_{\tau}^{-1 / \prime} H_{\min (C)}$. Then from the elementarity of $H_{\xi}$, pulled 
down by $\pi_{\tau}^{-1}$, it follows that for every $\xi \in \tau \cap C,\left(\pi_{\tau}^{-1 \prime \prime} H_{\xi}\right) \cap \mathcal{M}_{\alpha}$ is exactly equal to the hull of $\xi \cup p\left(\mathcal{M}_{\alpha}\right)$ in $\mathcal{M}_{\alpha}$.

Note that $\tau$ is regular relative to functions elementarily definable with parameters over $\mathcal{P}_{\tau}$. This is a consequence of Fact 3.10, since $\left(\tau^{+}\right)^{\mathcal{P}_{\tau}}=$ Ord $\cap \mathcal{R}_{\tau}$ has cofinality $\eta, \rho\left(\mathcal{P}_{\tau}\right)=\tau, \tau$ is regular in $\mathcal{P}_{\tau}$ (by the elementarity of $\pi_{\tau}$ and minimality of $\mathcal{P}_{\tau}$ ), and the cofinality of $\tau$ is different from $\eta$.

For each $\xi<\tau$, let $Y_{\xi}$ be the hull of $\xi \cup p\left(\mathcal{P}_{\tau}\right)$ in $\mathcal{P}_{\tau}$. Using the regularity established in the previous paragraph, it follows by the proof of Lemma 3.2 that for a club of $\xi<\tau, Y_{\xi} \cap \tau=\xi$ and the transitive collapse of $Y_{\xi}$ is an initial segment of $\mathcal{N}$ projecting to $\xi$. Since $\operatorname{Cof}(\tau) \notin\left\{\omega, \omega_{1}\right\}$ we can find such $\xi$ with $\xi \in C$ and $\operatorname{Cof}(\xi)$ uncountable. Since $\operatorname{Cof}(\tau) \neq \eta$ we can also ensure, by picking $\xi$ sufficiently large, that $\left\{\mathcal{M}_{\alpha} \mid \alpha \in A\right\} \subseteq Y_{\xi}$ for some $A$ cofinal in $\eta$. Then for $\alpha \in A, Y_{\xi} \cap \mathcal{M}_{\alpha}$ is exactly the hull of $\left(Y_{\xi} \cap \tau\right) \cup p\left(\mathcal{M}_{\alpha}\right)$ in $\mathcal{M}_{\alpha}$ by elementarity. By the calculations above and since $Y_{\xi} \cap \tau=\xi$, this hull is equal to $\left(\pi_{\tau}^{-1 \prime \prime} H_{\xi}\right) \cap \mathcal{M}_{\alpha}$. So $Y_{\xi} \cap \mathcal{M}_{\alpha}=\left(\pi_{\tau}^{-1 \prime \prime} H_{\xi}\right) \cap \mathcal{M}_{\alpha}$. Since $A$ is cofinal in $\eta$ it follows that $Y_{\xi} \cap \mathcal{R}_{\tau}=\pi_{\tau}^{-1 \prime \prime} H_{\xi}$.

Fix $\xi$ with the properties above, let $\overline{\mathcal{P}}$ be the transitive collapse of $Y_{\xi}$, and let $d: Y_{\xi} \rightarrow \overline{\mathcal{P}}$ be the collapse map. Since $Y_{\xi} \cap \mathcal{R}_{\tau}=\pi_{\tau}^{-1 "} H_{\xi}, d\left(\mathcal{R}_{\tau}\right)$ is exactly equal to $\mathcal{R}_{\xi}$. It follows that $\overline{\mathcal{P}}$ is a minimal premouse at or above $\mathcal{R}_{\xi}$ which projects to $\xi$. Since $\overline{\mathcal{P}}$ is a level of $\mathcal{N}$ this implies in particular that $\xi \in S$ and that $\overline{\mathcal{P}}=\mathcal{P}_{\xi}$. Moreover the fact that $Y_{\xi} \cap \mathcal{R}_{\tau}=\pi_{\tau}^{-1 \prime \prime} H_{\xi}$ also implies that $\pi_{\xi, \tau}=d^{-1} \uparrow \mathcal{R}_{\xi}$. Since all elements of $\mathcal{P}_{\tau}$ are definable over $\mathcal{P}_{\tau}$ from $\tau \cup \operatorname{range}\left(d^{-1}\right)$ it follows that $\mathcal{P}_{\tau}$ is equal to the ultrapower of $\overline{\mathcal{P}}=\mathcal{P}_{\xi}$ by the $(\xi, \tau)$ extender derived from $\pi_{\xi, \tau}$, namely $F_{\xi, \tau}$. In particular this ultrapower belongs to $\mathcal{N}$.

Claim 3.11. Let $\lambda$ be a regular uncountable cardinal. Let $\mathcal{N}$ be a weakly iterable premouse of ordinal height $\lambda$. Suppose $\lambda$ is a limit of cardinals of $\mathcal{N}$. Let $\mathcal{S}_{1}=\mathcal{S}(\mathcal{N})$, let $\gamma_{1}=\mathcal{S}_{1} \cap$ Ord, and suppose $\operatorname{Cof}\left(\gamma_{1}\right) \geq \lambda$. Suppose also that $\mathcal{S}_{1}$ has extenders indexed on its sequence cofinally in $\gamma_{1}$. Let $\mathcal{S}_{2}=\mathcal{S}\left(\mathcal{S}_{1}\right)$. Then there is no premouse $\mathcal{P} \unrhd \mathcal{S}_{2}$ so that all extenders on the sequence of $\mathcal{P}$ are on the sequence of $\mathcal{S}_{2}$ (where we consider top extenders, if they exist, to be on the sequence) and $\rho(\mathcal{P}) \leq \gamma_{1}$.

Proof. Suppose $\mathcal{P}$ is a counterexample to the claim. Let $\gamma_{2}=\mathcal{S}_{2} \cap$ Ord. We may assume, by passing to an initial segment of $\mathcal{P}$ if necessary, that no strict initial segment of $\mathcal{P}$ outside $\mathcal{S}_{2}$ projects to or below $\gamma_{1}$. Note $\mathcal{S}_{1}$ extends $\mathcal{N}$ strictly by the comment following Definition 3.4, and hence $\gamma_{1}>\lambda$ by Remark 3.8 .

It is enough to show that $\mathcal{P}$ is weakly iterable, and that every bounded subset of $\lambda$ which is definable over $\mathcal{P}$ from parameters, belongs to $\mathcal{P}$. The second part implies that $\rho(\mathcal{P}) \geq \lambda$. If $\rho(\mathcal{P})=\gamma_{1}$ it then follows from the first part that the core of $\mathcal{P}$ belongs to $U\left(\mathcal{S}_{1}\right)$, contradicting the fact that it extends $\mathcal{S}_{2}$. (Since every ordinal between $\lambda$ and $\gamma_{1}$ is collapsed to $\lambda$ in $\mathcal{P}$, functions on $\lambda$ into $\gamma_{1}$ can be coded by subsets of $\lambda$, definably over $\mathcal{P}$. Hence $\rho(\mathcal{P})=\gamma_{1}>\lambda$ implies that $\gamma_{1}$ is regular relative to functions that are elementarily definable over $\mathcal{P}$, as required in the definition of $U\left(\mathcal{S}_{1}\right)$.) If $\rho(\mathcal{P})=\lambda$ it follows that the core of $\mathcal{P}$ belongs to $U(\mathcal{N})$, contradicting the fact that it extends $\mathcal{S}_{1}$. 
Fix $\overline{\mathcal{P}}$ that embeds elementarily into $\mathcal{P}$, via $\sigma$ say. Suppose $|\overline{\mathcal{P}}|<\lambda$. We prove that $\overline{\mathcal{P}}$ embeds, via a map with the same critical point as $\sigma$, into a strict initial segment of a linear iterate of a strict initial segment of $\mathcal{S}_{1}$, by extenders indexed above $\lambda$. Applied with countable $\overline{\mathcal{P}}$, this establishes that $\mathcal{P}$ is weakly iterable, since every strict initial segment of $\mathcal{S}_{1}$ is weakly iterable, and weak iterability is inherited by linear iterates. Applied with $\overline{\mathcal{P}}$ equal to the hull in $\mathcal{P}$ of $\tau \cup a$ for $\tau<\lambda$ and finite $a \subseteq \mathcal{P}$, this establishes that all bounded subsets of $\lambda$ which are definable over $\mathcal{P}$ from parameters, belong to $\mathcal{P}$.

Recall from Andretta-Neeman-Steel [1, p. 166] that $\pi: \overline{\mathcal{M}} \rightarrow \mathcal{M}$ is a weak embedding if it is induced by a $\Sigma_{0}$ embedding from $\mathfrak{D}(\overline{\mathcal{M}})$ into $\mathfrak{D}(\mathcal{M})$. We say that $H \subseteq \mathcal{M}$ is a weak substructure of $\mathcal{M}$ if the anticollapse embedding from the transitive collapse of $H$ into $\mathcal{M}$ is a weak embedding.

We begin by defining a continuous $\subseteq$-increasing sequence $\left\langle H_{\alpha} \mid \alpha<\gamma_{1}\right\rangle$ of weak substructures of $\mathcal{P}$, with $\alpha \cup \operatorname{range}(\sigma) \subseteq H_{\alpha}$, and $\sup \left(H_{\alpha} \cap \gamma_{1}\right)<\gamma_{1}$.

If $k(\mathcal{P})>0$, let $\mathcal{Q}$ be the immediate truncation of $\mathcal{P}$. This is a premouse with the same universe, and $k(\mathcal{Q})=k(\mathcal{P})-1$. Let $H_{\alpha}$ be the elementary hull of $\alpha \cup \operatorname{range}(\sigma)$ in $\mathcal{Q}$. Then $H_{\alpha}$ is weak substructure of $\mathcal{P}$. For each finite $a \subseteq \operatorname{range}(\sigma)$, the hull of $\alpha \cup a$ in $\mathcal{Q}$ is bounded in $\gamma_{1}$, since $\rho(\mathcal{Q})>\gamma_{1}$. From this, the fact that $|\operatorname{range}(\sigma)|<\lambda$, and the assumption that $\operatorname{Cof}\left(\gamma_{1}\right) \geq \lambda$, it follows that $H_{\alpha}$ is bounded in $\gamma_{1}$.

If $k(\mathcal{P})=0$, let $H_{\alpha}$ be the elementary hull of $\alpha \cup \operatorname{range}(\sigma)$ in $\mathcal{P}\lceil\sup (\operatorname{range}(\sigma) \cap$ Ord). Then $H_{\alpha}$ is a weak substructure of $\mathcal{P}$, equivalently in this case $H_{\alpha}$ is $\Sigma_{0}$ elementary in $\mathcal{P}$. $H_{\alpha}$ is the union over $X \in \operatorname{range}(\sigma)$ and finite $a \subseteq \operatorname{range}(\sigma) \cap X$ of the hull of $\alpha \cup a$ in $X$. Using the facts that $|\operatorname{range}(\sigma)|<\lambda \leq \operatorname{Cof}\left(\gamma_{1}\right)$ and that $\gamma_{1}$ is a regular cardinal in $\mathcal{P}$, one can check that $H_{\alpha}$ is bounded in $\gamma_{1}$.

Now using the continuity of the sequence $\left\langle H_{\alpha} \mid \alpha<\gamma_{1}\right\rangle$, the fact that $H_{\alpha}$ is bounded below $\gamma_{1}$ for each $\alpha$, and the assumption that $\operatorname{Cof}\left(\gamma_{1}\right)$ is uncountable, fix some $\alpha<\gamma_{1}$ so that $\sup \left(H_{\alpha} \cap \gamma_{1}\right)=\alpha$. We may assume $\alpha>\lambda$.

Let $\hat{\mathcal{P}}$ be the transitive collapse of $H_{\alpha}$, and let $\pi: \hat{\mathcal{P}} \rightarrow \mathcal{P}$ be the anticollapse embedding. $\pi$ is a weak embedding. This implies that $\pi^{-1} \circ \sigma: \overline{\mathcal{P}} \rightarrow \hat{\mathcal{P}}$ is elementary: if a $\Sigma_{1}$ statement holds of $\left(\pi^{-1} \circ \sigma\right)(a)$ over $\mathfrak{D}(\hat{\mathcal{P}})$, witnessed by $b$ say, then since $\pi$ is weak, $\pi(b)$ witnesses that the same statement holds of $\sigma(a)$ over $\mathfrak{D}(\mathcal{P})$, hence by elementarity of $\sigma$ it holds of $a$ over $\mathfrak{D}(\overline{\mathcal{P}})$. Note further that $\operatorname{crit}\left(\pi^{-1} \circ \sigma\right)=\operatorname{crit}(\sigma)$, $\operatorname{since} \operatorname{crit}\left(\pi^{-1}\right)=\alpha>\operatorname{crit}(\sigma)$.

By definition of $\mathcal{S}_{2}$, there are cofinally many strict initial segments $\mathcal{M}$ of $\mathcal{S}_{2}$ which project to $\gamma_{1}$. For any such $\mathcal{M}$ which belongs to $H_{\alpha}, H_{\alpha} \cap \mathcal{M}$ is elementary in $\mathcal{M}$, and by condensation it is collapsed (by $\pi^{-1}$ ) to a strict initial segment of $\mathcal{S}_{1}$, in other words $\pi^{-1}(\mathcal{M}) \triangleleft \mathcal{S}_{1}$. It follows that $\pi^{-1}\left(\mathcal{S}_{2}\right)$ is an initial segment of $\mathcal{S}_{1}$ (possibly with its top extender removed). The initial segment is strict, since $\mathcal{S}_{1}$ has levels where it is seen that $|\alpha|=\lambda$, while $\pi^{-1}\left(\mathcal{S}_{2}\right)$ does not. By the claim assumptions it follows that there are extenders on the sequence of $\mathcal{S}_{1}$ indexed above $\pi^{-1}\left(\mathcal{S}_{2}\right)$. Let $\nu$ be the index of the first one.

$\hat{\mathcal{P}}$ is a premouse that extends $\pi^{-1}\left(\mathcal{S}_{2}\right)$ and has no extenders on its sequence beyond the ones occurring in $\pi^{-1}\left(\mathcal{S}_{2}\right)$. Hence $\hat{\mathcal{P}} \unlhd J_{\theta}\left(\pi^{-1}\left(\mathcal{S}_{2}\right)\right)$ for some $\theta$. From this and the conclusion of the previous paragraph it follows that $\hat{\mathcal{P}}$ is a strict initial segment of a linear iterate of $\mathcal{S}_{1} \|\langle\nu, 0\rangle$, obtained through iteratively taking 
the ultrapower of the current model by its first extender indexed at or above $\nu$, until reaching a model where the first such index is above $\theta$. Since $\overline{\mathcal{P}}$ embeds into $\hat{\mathcal{P}}$ via $\pi^{-1} \circ \sigma$, and $\operatorname{crit}\left(\pi^{-1} \circ \sigma\right)=\operatorname{crit}(\sigma)$, this completes the proof.

§4. Proof of Theorems 1.2 and 1.5. Fix $\delta$, assume $\mathrm{SBH}_{\delta}$, and suppose that $\delta$ is a Woodin cardinal. We will later add the remaining assumptions of Theorems 1.2 and 1.5 , but for now this is not necessary.

Let $\mathcal{W}$ be the model given by Lemma 2.4. Let $\mathcal{S}=\mathcal{S}(\mathcal{W})$. Let $\gamma=\operatorname{Ord} \cap \mathcal{S}$. By Claim 3.7, $\gamma>\delta, \gamma$ remains a cardinal in $L(\mathcal{S})$ and all subsets of $\delta$ in $L(\mathcal{S})$ belong to $\mathcal{S}$. By definition of $\mathcal{S}, \gamma=\left(\delta^{+}\right)^{L(\mathcal{S})}$. The class inner model we will work with is $L(\mathcal{S})$.

Claim 4.1. (Assuming $\delta$ is a Woodin cardinal, $\mathrm{SBH}_{\delta}, \mathcal{W}$ is given by Lemma 2.4, and $\gamma=\operatorname{Ord} \cap \mathcal{S}(\mathcal{W})$.) The cofinality of $\gamma$ is at least $\delta$.

Proof. Suppose $\operatorname{Cof}(\gamma)<\delta$. In particular $|\mathcal{S}|=\delta$. Let $\left\langle H_{\xi} \mid \xi<\delta\right\rangle$ be a continuous increasing chain with $\left|H_{\xi}\right|<\delta$ and $\bigcup_{\xi<\delta} H_{\xi}=\mathcal{S}$. Let $\mathcal{N}_{\xi}$ be the transitive collapse of $H_{\xi}$, and let $\sigma_{\xi}: \mathcal{N}_{\xi} \rightarrow \mathcal{S}$ be the anticollapse embedding. For $\xi<\zeta$ let $\sigma_{\xi, \zeta}=\sigma_{\zeta}^{-1} \circ \sigma_{\xi}$.

By Lemma 3.9 there is a club $C \subseteq \delta$, so that for every $\xi \in C$ with $\operatorname{Cof}(\xi) \notin$ $\left\{\omega, \omega_{1}, \operatorname{Cof}(\gamma)\right\}, \mathcal{N}_{\xi}$ is exactly equal to $\mathcal{W}\left\lceil\left(\xi^{+}\right)^{\mathcal{W}}\right.$. Thinning $C$ we may assume also that $H_{\xi} \cap \delta=\xi$ and $\sigma_{\xi}$ is elementary for all $\xi \in C$. Dropping an initial segment of $C$ if necessary we may assume $H_{\xi}$ is cofinal in $\gamma$, so $\sigma_{\xi}$ is cofinal into $\mathcal{S}$, and $\sigma_{\xi, \zeta}$ is cofinal into $\mathcal{N}_{\zeta}$.

Let $\overrightarrow{\mathcal{N}}=\left\langle\mathcal{N}_{\xi} \mid \xi<\delta\right\rangle$ and let $\vec{\sigma}=\left\langle\sigma_{\xi, \zeta} \mid \xi<\zeta<\delta\right\rangle$. Let $A \subseteq \delta$ code $\overrightarrow{\mathcal{N}}$, $\vec{\sigma}$, and $C$. Fix $\kappa \in \operatorname{Strong}(A)$. This is possible since $\delta$ is a Woodin cardinal. Note that $\kappa$ belongs to $C$ and has cofinality other than $\omega, \omega_{1}$, and $\operatorname{Cof}(\gamma)$ (in fact $\kappa$ is inaccessible, and certainly greater than $\left|\mathcal{N}_{0}\right|$ which is at least $\left.\operatorname{Cof}(\gamma)\right)$. Fix $\lambda>\kappa$ with the same properties. In particular then $\mathcal{N}_{\kappa}=\mathcal{W} \uparrow\left(\kappa^{+}\right)^{\mathcal{W}}$ and $\mathcal{N}_{\lambda}=\mathcal{W} \uparrow\left(\lambda^{+}\right)^{\mathcal{W}}$.

Let $\eta<\delta$ be an inaccessible cardinal above $\lambda$. Let $F^{*} \in V_{\delta}$ be an extender with critical point $\kappa$, which is $\eta$ strong relative to $\overrightarrow{\mathcal{N}}$ and $\vec{\sigma}$. Let $i_{F^{*}}$ be the ultrapower embedding by $F^{*}$, and let $\kappa^{*}=i_{F^{*}}(\kappa)$. By the strength of $F^{*}$ we have $i_{F^{*}}(\overrightarrow{\mathcal{N}})_{\lambda}=$ $\mathcal{N}_{\lambda}$ and $i_{F^{*}}(\vec{\sigma})_{\kappa, \lambda}=\sigma_{\kappa, \lambda}$. By the continuity of $\vec{H}, i_{F^{*}}(\vec{H})_{\kappa}=i_{F^{*}}{ }^{\prime \prime} H_{\kappa}$. It follows that $i_{F^{*}}(\overrightarrow{\mathcal{N}})_{\kappa}=\mathcal{N}_{\kappa}$ and $i_{F^{*}}(\vec{\sigma})_{\kappa, \kappa^{*}}=i_{F^{*}} \uparrow \mathcal{N}_{\kappa}$. Combining all this and the commutativity of the system $i_{F^{*}}(\vec{\sigma})$ we get that $i_{F^{*}}(\vec{\sigma})_{\lambda, \kappa^{*}} \circ \sigma_{\kappa, \lambda}=i_{F^{*}} \mid \mathcal{N}_{\kappa}$.

Let $F$ be the extender derived from the map $\sigma_{\kappa, \lambda}: \mathcal{N}_{\kappa} \rightarrow \mathcal{N}_{\lambda}$. Let $\alpha=$ $\left(\lambda^{+}\right)^{\mathcal{W}}=\operatorname{Ord} \cap \mathcal{N}_{\lambda}=\sup \sigma_{\kappa, \lambda}{ }^{\prime \prime}\left(\kappa^{+}\right)^{\mathcal{W}}$. Since $\mathcal{N}_{\kappa}=\mathcal{W}\left\lceil\left(\kappa^{+}\right)^{\mathcal{W}}, F\right.$ is an extender over $\mathcal{W}$. It maps cofinally into $\mathcal{W}\lceil\alpha$.

Let $h=i_{F^{*}}(\vec{\sigma})_{\lambda, \kappa^{*}}$. The critical point of $h$ is $\lambda$, and by our computations above, $h \circ F=i_{F^{*}}\left\lceil\mathcal{N}_{\kappa}\right.$.

Thus $F^{*}$ and $h$ witness the hypothesis of the maximality condition in Lemma 2.4 for $F$. It follows by the lemma that $F$ must be on the sequence of $\mathcal{W}$, indexed at $\alpha$. But then $\alpha$ is not a cardinal of $\mathcal{W}$, a contradiction.

The next two claims will use failure of $\square_{\delta}$, and threading for coherent sequences on $\delta$, to argue that $\delta$ is subcompact in $L(\mathcal{S})$. This will establish Theorem 1.2. The argument for this uses the results on $\square_{\delta}$ in Schimmerling-Zeman [11, 12], 
and is similar to the proofs in Section 4 of Jensen-Schimmerling-Schindler-Steel $[2]$.

Claim 4.2. Suppose $\square_{\delta}$ fails, and $\gamma=\delta^{+}$. Then $\delta$ is subcompact in $L(\mathcal{S})$.

Proof. If $\left(\delta^{+}\right)^{L(\mathcal{S})}=\left(\delta^{+}\right)^{V}$ then $\square_{\delta}$ reflects from $L(\mathcal{S})$ to $V$. Since $\square_{\delta}$ fails in $V$, it must fail in $L(\mathcal{S})$. Hence by Theorem 0.1 of Schimmerling-Zeman [12] applied in the fine structural model $L(\mathcal{S}), \delta$ is subcompact in $L(\mathcal{S})$.

Claim 4.3. Suppose $\delta$ is threadable, and $\operatorname{Cof}(\gamma)=\delta$. Then $\delta$ is subcompact in $L(\mathcal{S})$.

Proof. Suppose for contradiction that $\delta$ is not subcompact in $L(\mathcal{S})$. By Theorem 0.1 of Schimmerling-Zeman [12], $\square_{\delta}$ holds in $L(\mathcal{S})$. This is a combinatorial consequence of the existence of a $\square_{\delta}^{\prime}$ sequence on a club in $\gamma$, given by Theorem 3.1 of [12]. Let $\vec{C}=\left\langle C_{\alpha} \mid \delta<\alpha \in T\right\rangle$ be the canonical sequence witnessing this, constructed in Section 3 of [12]. (We use $T$ for the club $\mathcal{S}$ of [12], to avoid conflict with our own notation.)

Let $f: \delta \rightarrow \gamma$ be increasing, cofinal, and continuous. For $\alpha<\delta$ set $C_{\alpha}^{*}$ equal to $\left\{\xi<\alpha \mid f(\xi) \in C_{f(\alpha)}\right\}$ if this set is cofinal in $\alpha$, which must be the case if $\operatorname{Cof}(\alpha)>\omega$, and otherwise let $C_{\alpha}^{*}$ be a cofinal subset of $\alpha$ of order type $\leq \omega$. It is easy to check that $\left\langle C_{\alpha}^{*} \mid \alpha<\delta\right\rangle$ is coherent. By the assumptions of the claim it must have a thread, call it $D^{*}$. Then $f^{\prime \prime} D^{*}$ generates a thread through $\vec{C}$, call it $D$.

The definition of $\vec{C}$ in Schimmerling-Zeman [12] divides the ordinals in $T$ into two types. To avoid conflict with our own notation, we use $T^{0}$ and $T^{1}$ to denote the two types. These correspond to the sets $\mathcal{S}^{0}$ and $\mathcal{S}^{1}$ of [12]. The definition of $\vec{C}$ is such that for every $\tau \in T$ and $i \in\{0,1\}$, if $\tau \in T^{i}$ then $C_{\tau} \subseteq T^{i}$. It follows that our thread $D$ is either fully contained in $T^{0}$, or fully contained in $T^{1}$.

Schimmerling [10, Lemma 4.6] shows that (below superstrong) any thread through the canonical $\square_{\delta}$ sequence in a fine structural model leads to a premouse that collapses the successor of $\delta$ in the model. Our argument in the case $D \subseteq$ $T^{0}$ below is essentially the same, with the added notes that the restriction to below superstrong is not necessary, and that the resulting premouse is weakly iterable and projects exactly to $\delta$. Since this premouse extends $\mathcal{S}$ this leads to a contradiction. In the case $D \subseteq T^{1}$ below we use a different argument; we rule the case out using the fact that $\delta$ is a limit cardinal in $W$.

Following [12], let $\mathcal{N}_{\tau}$ for $\tau \in T$ be the collapsing structure for $\tau$ in our fine structural model, $L(\mathcal{S})$. Equivalently $\mathcal{N}_{\tau}$ is the collapsing structure for $\tau$ in $\mathcal{S}$, namely the least initial segment of $\mathcal{S}$ at or above $\mathcal{S} \uparrow \tau$ which projects to $\delta$. For $\tau \in T^{1}$ let $\mathcal{M}_{\tau}$ be the corresponding protomouse, defined on page 49 of [12]. We will say a bit more on the protomouse case later on.

Suppose first that $D \subseteq T^{0}$. In this case the definition in [12] is such that for any $\tau \in \operatorname{Lim}(D)$, and any sufficiently large $\bar{\tau} \in C_{\tau}=D \cap \tau$, there is an embedding $\sigma_{\bar{\tau}, \tau}: \mathcal{N}_{\bar{\tau}} \rightarrow \mathcal{N}_{\tau}$ which moves the standard parameter correctly and has generalized solidity witnesses for $p\left(\mathcal{N}_{\tau}\right)$ in its range, has critical point $\bar{\tau}$, sends $\bar{\tau}$ to $\tau$, and is induced by a $\Sigma_{0}$ preserving embedding of $\mathfrak{D}\left(\mathcal{N}_{\bar{\tau}}\right)$ into $\mathfrak{D}\left(\mathcal{N}_{\tau}\right)$.

It follows that such embeddings exist for all $\bar{\tau}<\tau$ both in $\hat{D}$, for some cofinal $\hat{D} \subseteq D$. Let $\mathcal{N}_{\infty}$ be the direct limit of the system $\left\langle\mathcal{N}_{\tau}, \sigma_{\bar{\tau}, \tau} \mid \bar{\tau}<\tau \in \hat{D}\right\rangle$. 
$\mathcal{N}_{\infty}$ extends $\mathcal{S}$, since $\mathcal{N}_{\tau}$ extends $\mathcal{S}\left\lceil\tau\right.$ for each $\tau$. $\mathcal{N}_{\infty}$ is weakly iterable, since every countable substructure of $\mathcal{N}_{\infty}$ embeds into $\mathcal{N}_{\tau}$ for any sufficiently large $\tau$ (as the cofinality of $\hat{D}$, which is equal to $\delta$, is uncountable). $\rho\left(\mathcal{N}_{\infty}\right)$ cannot be strictly smaller than $\delta$, since for any $\beta<\delta$, the $\Sigma_{1}$ theory of $\beta \cup p\left(\mathcal{N}_{\tau}\right)$ in $\mathfrak{D}\left(\mathcal{N}_{\tau}\right)$ stabilizes as $\tau \mapsto \gamma$ (using the fact that $\operatorname{Cof}(\gamma)=\delta$ ), and the stable value, being computable from $\mathcal{N}_{\tau}$ for any large enough $\tau$, belongs to $\mathcal{S}$. On the other hand the $\Sigma_{1}$ theory of $\delta \cup p\left(\mathcal{N}_{\infty}\right)$ in $\mathfrak{D}\left(\mathcal{N}_{\infty}\right)$ cannot belong to $\mathcal{S}$, since it would then belong to $\mathcal{N}_{\tau}$ for all sufficiently large $\tau$, but by the preservation properties of the embeddings it subsumes, and hence computes, the $\Sigma_{1}$ theory of $\delta \cup p\left(\mathcal{N}_{\tau}\right)$ in $\mathfrak{D}\left(\mathcal{N}_{\tau}\right)$, which does not belong to $\mathcal{N}_{\tau}$.

In conclusion then $\mathcal{N}_{\infty}$ is a weakly iterable premouse which extends $\mathcal{S}$ and projects to $\delta$. But this contradicts the definition of $\mathcal{S}$.

Suppose next that $D \subseteq T^{1}$. In this case the construction in SchimmerlingZeman [12] is such that for every $\tau \in \operatorname{Lim}(D)$ and all sufficiently large $\bar{\tau} \in C_{\tau}=$ $D \cap \tau$, there is an embedding $\sigma_{\bar{\tau}, \tau}$ between the protomice $\mathcal{M}_{\bar{\tau}}$ and $\mathcal{M}_{\tau}$. The precise condition can be found on page 51 of [12]. What is important for us is that each protomouse $\mathcal{M}_{\tau}$ has a top predicate $F_{\tau}$ which is a partial extender over $\mathcal{M}_{\tau}$, that $\operatorname{crit}\left(F_{\bar{\tau}}\right)$ and $\operatorname{crit}\left(F_{\tau}\right)$ are both below $\delta$, that $\sigma_{\bar{\tau}, \tau}$ fixes $\operatorname{crit}\left(F_{\bar{\tau}}\right)$, and that the domain of $F_{\tau}$ is strictly larger than the domain of $F_{\bar{\tau}}$. We can as before restrict to a cofinal $\hat{D} \subseteq D$ so that these conditions hold for all $\bar{\tau}<\tau$ both in $\hat{D}$. Let $\mu=\operatorname{crit}\left(F_{\tau}\right)$ for some, and equivalently for all, $\tau \in \hat{D}$. For each $\tau \in \hat{D}$, the domain of $F_{\tau}$ is a strict subset of the powerset of $\mu$ in $\mathcal{S}$, equivalently in $\mathcal{W}$ as $\mu<\delta$. But the conditions above are such that the domains of $F_{\tau}$, for $\tau \in \hat{D}$, are strictly increasing as $\tau \mapsto \gamma$. Since $\operatorname{Cof}(\gamma)=\delta$, and since the powerset of $\mu$ in $\mathcal{W}$ has size smaller than $\delta$, this is a contradiction.

Since $\gamma \leq \delta^{+}$, and since $\operatorname{Cof}(\gamma) \geq \delta$ by Claim 4.1, the last two claims establish Theorem 1.2 .

For the proof of Theorem 1.5 we need the following results, which allow characterizing, in fine structural inner models, the statement that $\delta^{+}$is threadable in terms of $\Pi_{1}^{2}$ subcompactness. A related characterization in terms of failure of simultaneous stationary reflection was obtained earlier by Kypriotakis-Zeman [3]. The proof of our characterization, and the coherent sequence we construct during the proof, are both very similar to those in [3].

LEMma 4.4. Let $\mathcal{Q}$ be a weakly iterable premouse. Let $\gamma$ be a successor cardinal in $\mathcal{Q}$, say $\gamma=\left(\delta^{+}\right)^{\mathcal{Q}}$. Suppose that $\delta$ is not $\Pi_{1}^{2}$ subcompact in $\mathcal{Q}$. Then there exists a coherent sequence $\vec{C}=\left\langle C_{\alpha} \mid \alpha<\gamma\right\rangle$ in $\mathcal{Q}$, so that from any thread through $\vec{C}$ one can continuously and definably over $(\mathcal{Q} \mid \gamma ; \vec{C})$ obtain either:

1. A function witnessing that $\operatorname{Cof}^{V}(\gamma)$ is smaller than $\delta$;

2. A length $\gamma$ directed system of weakly iterable premice, whose direct limit extends $\mathcal{Q}\lceil\gamma$ and projects to $\delta$; or

3. A length $\gamma$ directed system of weakly iterable premice whose direct limit extends $\mathcal{Q} \mid \gamma$, satisfies that $\gamma$ is the largest cardinal, and has a subset of $\gamma$ which does not belong to $\mathcal{Q}$.

Proof. To define the coherent sequence we work in $\mathcal{Q}$. Fix a $\Pi_{1}^{1}$ formula $(\forall X) \varphi(A, X)$ which, together with some $A \subseteq \gamma$, witnesses that $\delta$ is not $\Pi_{1}^{2}$ 
subcompact. Let $\psi(A, \gamma)$ be the statement that $(\forall X) \varphi(A, X)$ and $A$ witness failure of $\Pi_{1}^{2}$ subcompactness. $\psi$ is a $\Pi_{1}$ formula in $A$ and $\gamma$ over $\mathcal{Q}$. Let $A$ be least, in the canonical wellordering $<_{\mathcal{Q}}$ of $\mathcal{Q}$, so that $\psi(A, \gamma)$ holds. Let $\overline{\mathcal{Q}}$ be the first level of $\mathcal{Q}$ so that $\gamma, A \in \overline{\mathcal{Q}}$ and the $\Sigma_{1}$ statement $\left(\forall Z<_{\mathcal{Q}} A\right)(Z \subseteq \gamma \rightarrow$ $\neg \psi(Z, \gamma))$ holds in $\overline{\mathcal{Q}}$. Note that $\rho(\overline{\mathcal{Q}})=\gamma=\delta^{+}$.

For each $\nu<\delta^{+}$, let $\overline{\mathcal{Q}}_{\nu}$ be the transitive collapse of the hull of $\nu \cup p(\overline{\mathcal{Q}})$ in $\overline{\mathcal{Q}}$. Let $j_{\nu}: \overline{\mathcal{Q}}_{\nu} \rightarrow \overline{\mathcal{Q}}$ be the anticollapse embedding. Then there is a club $C \subseteq \delta^{+}$so that for every $\nu \in C, \operatorname{crit}\left(j_{\nu}\right)=\nu, j_{\nu}(\nu)=\delta^{+}, A \in \operatorname{range}\left(j_{\nu}\right)$, every subset of $\delta$ definable over $\overline{\mathcal{Q}}$ from parameters in range $\left(j_{\nu}\right)$ belongs to $\overline{\mathcal{Q}}_{\nu}$, and every element of range $\left(j_{\nu}\right)$ differs from the $\Sigma_{1}$ theory of $\delta^{+} \cup p(\overline{\mathcal{Q}})$ in $\overline{\mathcal{Q}}$ on some tuple from $\nu \cup p(\overline{\mathcal{Q}}) . \overline{\mathcal{Q}}_{\nu}$ projects to $\nu$, extends $\mathcal{Q} \mid \nu$, and its largest cardinal below $\nu$ is $\delta$. By condensation, for every $\nu \in C$ one of the following holds:

i. $\overline{\mathcal{Q}}_{\nu}$ is an initial segment of $\mathcal{Q}$ (and in particular $E_{\nu}^{\mathcal{Q}}=\emptyset$ ); or

ii. $E_{\nu}^{\mathcal{Q}} \neq \emptyset$, $\operatorname{crit}\left(E_{\nu}^{\mathcal{Q}}\right)<\delta$, and $\overline{\mathcal{Q}}_{\nu}$ is an initial segment of $\operatorname{Ult}_{0}\left(\mathcal{Q}, E_{\nu}^{\mathcal{Q}}\right)$.

Claim 4.5. If condition (ii) holds, then the $\Sigma_{1}^{1}$ statement $(\exists X) \neg \varphi(A \cap \nu, X)$ holds in $\operatorname{Ult}_{0}\left(\mathcal{Q}, E_{\nu}^{\mathcal{Q}}\right)$ over $\mathcal{Q}\lceil\nu$.

Proof. Let $F=E_{\nu}^{\mathcal{Q}}$ and let $\kappa=\operatorname{crit}(F) . \operatorname{Ult}_{0}(\mathcal{Q}, F)$ and $\mathcal{Q}$ agree to $\nu$, and $\nu$ is the successor of $\delta$ in the ultrapower. Suppose for contradiction that $(\forall X) \varphi(A \cap \nu, X)$ holds in $\operatorname{Ult}_{0}(\mathcal{Q}, F)$ over $\mathcal{Q}\lceil\nu$. This implies that $\psi(A \cap \nu, \nu)$ holds in the ultrapower, meaning that the $\Pi_{1}^{1}$ statement $(\forall X) \varphi(A \cap \nu, X)$ and $A \cap \nu$ witness failure of $\Pi_{1}^{2}$ subcompactness of $\delta$ in $\operatorname{Ult}_{0}(\mathcal{Q}, F)$, since any subcompactness embedding into $\overline{\mathcal{Q}}_{\nu} \uparrow \nu$ can be composed with $j_{\nu}$ to produce a subcompactness embedding into $\mathcal{Q} \mid \gamma$. By elementarity of $j_{\nu}$ and since $\overline{\mathcal{Q}}_{\nu}$ is an initial segment of $\operatorname{Ult}_{0}(\mathcal{Q}, F), A \cap \nu$ must in fact be least so that $\psi(A \cap \nu, \nu)$ holds in $\operatorname{Ult}_{0}(\mathcal{Q}, F)$. It follows in particular that $A \cap \nu$ is definable in the ultrapower from $\nu$. Since $\nu$ belongs to the range of the ultrapower embedding $i_{F}$, so does $A \cap \nu$.

Let $B \subseteq \kappa^{+}$be such that $i_{F}(B)=A \cap \nu$. By elementarity of $i_{F}$, the $\Pi_{1}^{1}$ statement $(\forall X) \varphi(B, X)$ holds in $\mathcal{Q}$ over $\mathcal{Q}\left\lceil\kappa^{+}\right.$. But then the embedding $j_{\nu} \circ F:\left(\mathcal{Q} \mid \kappa^{+} ; \kappa, B\right) \rightarrow(\mathcal{Q} \mid \gamma ; \delta, A)$ witnesses the instance of $\Pi_{1}^{2}$ subcompactness corresponding to $(\forall X) \varphi(A, X)$, contradicting the choice of $\varphi$ and $A$. $\dashv$

Lemma 4.4 calls for a coherent sequence on $\gamma$. But it is enough to construct a club $C \subseteq \gamma$ and a sequence $\left\langle C_{\alpha} \mid \alpha \in C\right\rangle$ with the following properties: $C_{\alpha} \subseteq C \cap \alpha$ is closed below $\alpha ; \beta \in C_{\alpha} \rightarrow C_{\beta}=C_{\alpha} \cap \beta$; and if $\alpha$ is a limit point of $C$ of uncountable cofinality, then $C_{\alpha}$ is unbounded in $\alpha$. We refer to such sequences as weak coherent sequences. Any weak coherent sequence $\vec{C}$ can be converted to a coherent sequence $\vec{C}^{\prime}=\left\langle C_{\alpha}^{\prime} \mid \alpha<\delta\right\rangle$ by letting $C_{\alpha}^{\prime}=f^{-1 / \prime} C_{f(\alpha)}$ where $f: \gamma \rightarrow C$ is order preserving and continuous, and then replacing any $C_{\alpha}^{\prime}$ which is bounded in $\alpha$ by a cofinal subset of $\alpha$ of order type $\leq \omega$. Threads through $\vec{C}^{\prime}$ can be continuously converted to threads through $\vec{C}$.

We define a weak coherent sequence $\vec{C}$. The sequence splits into distinct components which do not interact. On one component, the set $I$ below, we will use witnesses to the conclusion of Claim 4.5. By the claim any $\nu$ not covered by the first component must fall under condition (i) above, meaning that it does not index an extender in $\mathcal{Q}$. On such $\nu$ we will use the definitions from the $\square_{\delta}$ construction of Schimmerling-Zeman [12]. 
Let $I \subseteq C$ be the set of all $\nu \in C$ for which there exists an initial segment $\mathcal{M}$, of $\mathcal{Q}$ if $E_{\nu}^{\mathcal{Q}}=\emptyset$ and of $\operatorname{Ult}_{0}\left(\mathcal{Q}, E_{\nu}^{\mathcal{Q}}\right)$ if $E_{\nu}^{\mathcal{Q}} \neq \emptyset$, which extends $\mathcal{Q}\lceil\nu$, satisfies that $\nu$ is the largest cardinal, does not project below $\nu$, has $A \cap \nu$ as an element, and satisfies " $(\exists X) \neg \varphi(A \cap \nu, X)$ holds over $\mathcal{Q} \backslash \nu$ ". For $\nu \in I$ let $\mathcal{M}_{\nu}$ be the least premouse $\mathcal{M}$ witnessing this.

Note that $\rho\left(\mathcal{M}_{\nu}\right)=\nu$. For $\alpha<\nu$ let $H_{\alpha}^{\nu}$ be the hull of $\alpha \cup p\left(\mathcal{M}_{\nu}\right) \cup\{A \cap \nu\}$ in $\mathcal{M}_{\nu}$. Let $C_{\nu}$ be the set of $\alpha \in C \cap \nu$ so that $H_{\alpha}^{\nu} \cap \nu=\alpha$, and every subset of $\delta$ which is definable over $\mathcal{M}_{\nu}$ from parameters in $H_{\alpha}^{\nu}$ belongs to $H_{\alpha}^{\nu}$. It is clear that $C_{\nu}$ is closed. By condensation, for any $\alpha \in C_{\nu}$, the transitive collapse of $H_{\alpha}^{\nu}$ is a structure satisfying the conditions in the previous paragraph. It follows that $C_{\nu} \subseteq I$. Using the minimality of $\mathcal{M}_{\alpha}$ it also follows that the transitive collapse of $H_{\alpha}^{\nu}$ is exactly $\mathcal{M}_{\alpha}$. From this and the definitions it is clear then that $C_{\alpha}=C_{\nu} \cap \alpha$.

For every $\beta<\nu, \sup \left(H_{\beta}^{\nu} \cap \nu\right)<\nu$, since otherwise $H_{\beta}^{\nu} \supseteq \nu$, and this implies that $H_{\beta}^{\nu}=\mathcal{M}_{\nu}$, contradicting the fact that $\rho\left(\mathcal{M}_{\nu}\right)>\delta$. Again using the fact that $\rho\left(\mathcal{M}_{\nu}\right)>\delta$, the theory of $\beta \cup p\left(\mathcal{M}_{\nu}\right) \cup\{A \cap \nu\}$ in $\mathcal{M}_{\nu}$ belongs to $\mathcal{M}_{\nu}$. If $\nu$ has uncountable cofinality we may therefore find a club of limit $\alpha<\nu$ so that for arbitrarily large $\beta<\alpha, \sup \left(H_{\beta}^{\nu} \cap \nu\right)$ and the theory of $\beta \cup p\left(\mathcal{M}_{\nu}\right) \cup\{A \cap \nu\}$ in $\mathcal{M}_{\nu}$ both belong to $H_{\alpha}^{\nu}$. Any such $\alpha$ in $C$ then belongs to $C_{\nu}$. So $C_{\nu}$ is unbounded in $\nu$ whenever $\nu$ is a limit point of $C$ of uncountable cofinality.

So far we defined $C_{\nu}$ for $\nu \in I$, satisfying the requirements of weak coherence, and moreover with the property that $C_{\nu} \subseteq I$ for $\nu \in I$. Let $J=C-I$. By Claim 4.5 and the definition of $I$, any $\nu \in J$ must fall under condition (i) above. In particular $\nu$ does not index an extender in $E^{\mathcal{Q}}$. This allows us to use the definitions from the construction of the $\square_{\delta}$ sequence in Schimmerling-Zeman [12]. Let $J^{0}, J^{1}$ be the partition of $J$ into the two types defined in [12]. Let $\mathcal{N}_{\nu}$ be the collapsing structure for $\nu$ in $\mathcal{Q}$. For $\nu \in J^{1}$ let $\mathcal{M}_{\nu}$ be the associated protomouse. Let $C_{\nu}$ be the $\square_{\delta}^{\prime}$ set for $\nu$ given by the construction in the proof of Theorem 3.1 of [12]. $C_{\nu}$ is contained in $J^{0}$ if $\nu \in J^{0}$, and contained in $J^{1}$ if $\nu \in J^{1}$.

For $\nu \in J$ and $\alpha \in C_{\nu}$ there is a $\Sigma_{0}$ elementary embeddings from $\mathcal{N}_{\alpha}$ into $\mathcal{N}_{\nu}$ if $\nu \in J^{0}$, and from $\mathcal{M}_{\alpha}$ into $\mathcal{M}_{\nu}$ if $\nu \in J^{1}$. This implies in particular that $\mathcal{N}_{\alpha}$ (respectively $\mathcal{M}_{\alpha}$ ) has no strict initial segment $\mathcal{M}$ that satisfies the conditions in the definition of $I$ for $\alpha$, since any such segment would be pushed to a strict initial segment of $\mathcal{N}_{\nu}$ (respectively $\mathcal{M}_{\nu}$ ) with the same properties, and this would contradict the fact that $\nu \notin I$. Since any $\mathcal{M}$ satisfying the conditions in the definition of $I$ for $\alpha$ must occur before $\alpha$ is collapsed to $\delta$, it follows that $\alpha \notin I$. Hence $C_{\alpha}$ too is defined following [12], and using the weak coherence proved in [12] it follows that $C_{\alpha}=C_{\nu} \cap \alpha$.

This completes the definition of the weak coherent sequence $\vec{C}$. Suppose now that $D$ is a thread through $\vec{C}$. Our definition of $\vec{C}$ is such that $\alpha \in I \rightarrow C_{\alpha} \subseteq I$, $\alpha \in J^{0} \rightarrow C_{\alpha} \subseteq J^{0}$, and $\alpha \in J^{1} \rightarrow C_{\alpha} \subseteq J^{1}$. Thus $D$ must be entirely contained in one of $I, J^{0}, J^{1}$.

If $D \subseteq J^{1}$, then the argument at the end of the proof of Claim 4.3 shows that the top extenders of the protomice $\mathcal{M}_{\alpha}$ for $\alpha \in D$ have increasing domains that are nonetheless bounded below $\delta$. The sequence $\left\langle\mathcal{M}_{\alpha} \mid \alpha \in D\right\rangle$ then gives 
rise to an order preserving embedding of $D$ into an ordinal below $\delta$, witnessing condition (1) of Lemma 4.4.

If $D \subseteq J^{0}$, then the main argument in the proof of Claim 4.3 shows that the direct limit of the premice $\left\langle\mathcal{N}_{\alpha} \mid \alpha \in D\right\rangle$ extends $\mathcal{Q}\lceil\gamma$ and projects to $\delta$. This gives condition (2) of Lemma 4.4.

Finally, assume $D \subseteq I$. For each $\alpha<\nu$ both in $I, \mathcal{M}_{\alpha}$ is the transitive collapse of the hull $H_{\alpha}^{\nu}$. Let $\mathcal{M}^{*}$ be the direct limit of the premice $\left\langle\mathcal{M}_{\alpha} \mid \alpha \in D\right\rangle$ under the anticollapse embeddings. It is clear that $\mathcal{M}^{*}$ extends $\mathcal{Q} \mid \gamma, A \in \mathcal{M}^{*}$, and using elementarity of the embeddings, $\gamma$ is the largest cardinal in $\mathcal{M}^{*}$. The direct limit embedding of $\mathcal{M}_{\alpha}$ into $\mathcal{M}^{*}$ sends $A \cap \alpha$ to $A$, and $\alpha$ to $\gamma$. Using elementarity and the definition of $I$ and $\mathcal{M}_{\alpha}$, it follows that $\mathcal{M}^{*}$ satisfies " $(\exists X) \neg \varphi(A, X)$ holds over $\mathcal{Q} \mid \gamma$ ". By our choice of $\varphi$ and $A$ this statement fails in $\mathcal{Q}$. So $\mathcal{M}^{*}$ has a subset of $\mathcal{Q} \uparrow \gamma$ that does not belong to $\mathcal{Q}$, and this gives condition (3) of Lemma 4.4 .

THEOREM 4.6. Let $\mathcal{Q}$ be a weakly iterable premouse. Let $\delta^{+}$be a successor cardinal of $\mathcal{Q}$. Then in $\mathcal{Q}, \delta$ is $\Pi_{1}^{2}$ subcompact iff $\delta^{+}$is threadable.

Proof. The left-to-right direction is given by Lemma 1.4. Suppose, for the right-to-left direction, that in $\mathcal{Q}, \delta^{+}$is threadable, yet $\delta$ is not $\Pi_{1}^{2}$ subcompact. Let $\vec{C} \in \mathcal{Q}$ be a coherent sequence witnessing Lemma 4.4. By assumption there is a thread $D$ through $\vec{C}$ in $\mathcal{Q}$. By Lemma 4.4 one can obtain from this thread a set which does not belong to $Q$. (This set is either a witness that $\delta^{+}$is singular, or a subset of $\delta$ outside $\mathcal{Q}$, or a subset of $\delta^{+}$outside $\mathcal{Q}$.) But this is a contradiction since $D \in \mathcal{Q}$.

With Lemma 4.4 at hand we can now prove Theorem 1.5. Fix a Woodin cardinal $\delta$. Assume $\mathrm{SBH}_{\delta}$. Suppose that $\delta$ and $\delta^{+}$are both threadable.

Let $\mathcal{W}$ be the model given by Lemma 2.4 . Let $\mathcal{S}_{1}=\mathcal{S}(\mathcal{W})$. Let $\gamma_{1}=\operatorname{Ord} \cap \mathcal{S}_{1}$. By Claim 4.1, $\operatorname{Cof}\left(\gamma_{1}\right) \geq \delta$. Using Lemma 3.2 then the stack $\mathcal{S}\left(\mathcal{S}_{1}\right)$ is well defined. Let $\mathcal{S}_{2}=\mathcal{S}\left(\mathcal{S}_{1}\right)$ and let $\gamma_{2}=\operatorname{Ord} \cap \mathcal{S}_{2}$.

Our proof of Theorem 1.2 shows that $\gamma_{1}=\left(\delta^{+}\right)^{L\left(\mathcal{S}_{1}\right)}$, and $\delta$ is subcompact in $L\left(\mathcal{S}_{1}\right)$. In particular there are extenders on the sequence of $\mathcal{S}_{1}$ indexed cofinally in $\gamma_{1}$. We can therefore apply Claim 3.11. It follows from the claim that $\gamma_{2}>\gamma_{1}$, and that $\gamma_{1}$ and $\gamma_{2}$ remain cardinals in $L\left(\mathcal{S}_{2}\right)$. Hence $\gamma_{1}$ and $\gamma_{2}$ are respectively the successor and double successor of $\delta$ in $L\left(\mathcal{S}_{2}\right)$, and $H\left(\gamma_{1}\right)^{L\left(\mathcal{S}_{2}\right)}=\mathcal{S}_{1}$.

We prove that $\delta$ is $\Pi_{1}^{2}$ subcompact in $L\left(\mathcal{S}_{2}\right)$. Suppose for contradiction that this is not the case. Let $\vec{C} \in L\left(\mathcal{S}_{2}\right)$ be the coherent sequence given by Lemma 4.4. This is a coherent sequence on $\gamma_{1}$, which is either equal to $\delta^{+}$or has cofinality $\delta$, by Claim 4.1. Either way $\vec{C}$ has a thread (in $V$ ), since we are assuming both $\delta$ and $\delta^{+}$are threadable.

Let $D$ be a thread through $\vec{C}$. Then one of the three cases of Lemma 4.4 must hold. The first is impossible since $\operatorname{Cof}\left(\gamma_{1}\right) \geq \delta$. For the second and third, note that since $\operatorname{Cof}\left(\gamma_{1}\right) \geq \delta>\omega$, weak iterability transfers from individual models to the direct limit, in any directed system of length $\gamma_{1}$. So condition (2) of the lemma gives a sound weakly iterable premouse extending $\mathcal{S}_{1}$ and projecting to $\delta$, while condition (3) gives a sound weakly iterable premouse extending $\mathcal{S}_{1}$ which defines a subset of $\gamma_{1}$ that does not belong to $\mathcal{S}_{2}$, without collapsing $\gamma_{1}$ in its rudimentary closure. But by definitions of $\mathcal{S}_{1}$ and $\mathcal{S}_{2}$ this premouse belongs to $\mathcal{S}_{1}$ 
in the former case and to $\mathcal{S}_{2}$ in the latter, giving a contradiction. This completes the proof of Theorem 1.5.

§5. Long extenders. Our goal in this section is to present some of the main ideas in the proof of Theorem 1.9. We will be vague about what we mean by a long extender premouse. In broad terms this is a premouse where the coherence condition is modified in the natural way to allow long extenders. But one crucial additional property that we place is that, for a structure $(\mathcal{M} ; F)$ to be coherent, the first projectum of the structure must be equal to or smaller than $F(\operatorname{crit}(F)$ ). (We also place some conditions on the parameter used for the projectum, intended to compensate for lack of solidity of the parameter, which we allow in some cases. Another condition related to compensating for lack of solidity is amenable closure of the premouse for some definable subsets. But we will not get to these issues here.) With this requirement, whenever $\nu$ is the index of a long extender in a long extender premouse $\mathcal{M}, \mathcal{M} \|\langle\nu, 0\rangle$ projects to or below $E_{\nu}^{M}\left(\operatorname{crit}\left(E_{\nu}^{M}\right)\right)$.

There are several reasons for placing this requirement. The main one is that it allows us to argue that comparisons terminate even if "long" generators are moved.

Let $\mathcal{P}$ be a long extender premouse, and let $\mathcal{T}$ be an iteration tree on $\mathcal{P}$, with models $\mathcal{P}_{\xi}$, embeddings $i_{\xi, \zeta}$, and extenders $F_{\xi}$ say. $\mathcal{T}$ is non-overlapping if for every successor ordinal $\epsilon+1<\operatorname{lh}(\mathcal{T})$, the $<_{\mathcal{T}}$ predecessor of $\epsilon+1$ is the least $\xi$ so that $\operatorname{crit}\left(F_{\epsilon}\right)<F_{\xi}\left(\operatorname{crit}\left(F_{\xi}\right)\right)$. This is precisely the same as the definition used in the case of short extender premice. It allows long generators on the branch leading to $\mathcal{P}_{\xi}$ to be moved by the embedding $i_{\xi, \epsilon+1}$.

When comparing countable long extender premice $\mathcal{M}$ and $\mathcal{N}$, we form iteration trees which are non-overlapping in the above sense. Assuming, for contradiction, that the comparison runs to $\omega_{1}$, and letting $i_{\xi, \omega_{1}}: \mathcal{M}_{\xi} \rightarrow \mathcal{M}_{\omega_{1}}$ and $j_{\xi, \omega_{1}}: \mathcal{N}_{\xi} \rightarrow$ $\mathcal{N}_{\omega_{1}}$ be the direct limit embeddings on the two sides of the comparison, we have as usual some $\alpha<\omega_{1}$ so that $i_{\alpha, \omega_{1}}$ and $j_{\alpha, \omega_{1}}$ agree on the common part of their domains. Letting $F$ and $G$ be the first extenders used along the branches $\left[\alpha, \omega_{1}\right]$ on the two sides, and letting $\beta$ and $\gamma$ be the indices of the models these extenders are taken from, we have that $i_{\alpha, \omega_{1}}$ extends $i_{\beta+1, \omega_{1}} \circ F$, and $j_{\alpha, \omega_{1}}$ extends $j_{\gamma+1, \omega_{1}} \circ$ $G$. Assuming for simplicity that $F$ and $G$ have the same domain, it follows from the agreement between $i_{\alpha, \omega_{1}}$ and $j_{\alpha, \omega_{1}}$ that $i_{\beta+1, \omega_{1}} \circ F=j_{\gamma+1, \omega_{1}} \circ G$.

Since the trees on the two sides are non-overlapping, $\operatorname{crit}\left(i_{\beta+1, \omega_{1}}\right) \geq F(\operatorname{crit}(F))$ and $\operatorname{crit}\left(j_{\gamma+1, \omega_{1}}\right) \geq G(\operatorname{crit}(G))$. If $F$ and $G$ were short we could now argue that they are compatible, and this would lead to a contradiction. We cannot take this route here since generators of $F$ at or above $F(\operatorname{crit}(F))$ are moved by $i_{\beta+1, \omega_{1}}$, and similarly on the other side of the comparison.

Let $\mu$ be the index of $F$ in $\mathcal{M}_{\beta}$, and let $\nu$ be the index of $G$ in $\mathcal{N}_{\gamma}$. Let $\overline{\mathcal{M}}=\mathcal{M}_{\beta} \|\langle\mu, 0\rangle$ and $\overline{\mathcal{N}}=\mathcal{N}_{\gamma} \|\langle\nu, 0\rangle$. These are active long extender premice, with their top predicates equal to the extenders $F$ and $G$ respectively. The maps $i_{\beta+1, \omega_{1}}$ and $j_{\gamma+1, \omega_{1}}$ can be used to stretch these two structures. Let $\mathcal{M}^{*}$ and $\mathcal{N}^{*}$ be the resulting structures. Both are long extender premice, with top predicates $F^{*}$ and $G^{*}$ which are equal to $i_{\beta+1, \omega_{1}} \circ F$ and $j_{\gamma+1, \omega_{1}} \circ G$ respectively. By the conclusion of the paragraph before last, $F^{*}=G^{*}$, and hence in fact $\mathcal{M}^{*}=\mathcal{N}^{*}$. 
By our requirement on projecta of active stages, $\rho(\overline{\mathcal{M}}) \leq F(\operatorname{crit}(F))$. From this, the fact that $\operatorname{crit}\left(i_{\beta+1, \omega_{1}}\right) \geq F(\operatorname{crit}(F))$, and preservation of a canonically chosen parameter $p(\overline{\mathcal{M}})$, we can conclude that $\overline{\mathcal{M}}$ is equal to the transitive collapse of the hull of $F(\operatorname{crit}(F)) \cup p\left(\mathcal{M}^{*}\right)$ in $\mathcal{M}^{*}$. The same is true on the other side of the comparison.

Since $\mathcal{M}^{*}=\mathcal{N}^{*}$, this allow us to argue that $\overline{\mathcal{M}}=\overline{\mathcal{N}}$ in case both are sound, and hence in particular $F=G$, contradicting the fact that $F$ and $G$ were used in a comparison. In effect what is happening here is that we use soundness and low projectum to argue that the long generators of $F$ and $G$ are moved in exactly the same way on the two sides, and this leads to a contradiction.

In case $\overline{\mathcal{M}}$ and $\overline{\mathcal{N}}$ are not both sound, one has to go a little deeper into fine structural arguments, but still the fact that both are transitive collapses of hulls of $\mathcal{M}^{*}=\mathcal{N}^{*}$ leads to a contradiction.

We omitted many details in the sketch above. Most important among them involve the way we make sure that canonical parameters in $\overline{\mathcal{M}}$ and $\overline{\mathcal{N}}$ are moved correctly by the embeddings into $\mathcal{M}^{*}=\mathcal{N}^{*}$. We cannot use solidity for this, since termination of the comparison process is a prerequisite for the solidity proof. In fact in some cases the parameters we pick are not solid. Still, with some additional restrictions on premice we can argue that they are moved correctly. The details can be found in [7].

We turn now to the construction of long extender premice. Our restriction that active stages with top predicate $F$ must project to or below $F(\operatorname{crit}(F))$ may seem a drastic limitation on the extenders in our models. But notice that, in a premouse $\mathcal{M}$ say, this restriction only limits the extenders that are on the sequence $\vec{E}^{\mathcal{M}}$. We will see that it does not prevent us from having other extenders $F$, which do not define any new subsets of $F(\operatorname{crit}(F))$, as elements of $\mathcal{M}$ which are not on the sequence $\vec{E}^{\mathcal{M}}$.

REMARK 5.1. The insight that for inner model theory at the level of supercompact cardinals one can gain substantially by arguing that extenders are put into models not because they are on the sequence of the model, but because they can be coded by tuples of extenders from the sequence, is due to Woodin $[17,18]$. The structure of the comparison argument above was first discovered by Steel in the more limited setting of extenders with few generators, meaning extenders $F$ with at most $F(\operatorname{crit}(F))$ generators. Woodin then realized that the key to this is that $F$ projects to $F(\operatorname{crit}(F))$. The argument was written up in Steel [14]. Feng and Woodin (2002, unpublished) used these comparison ideas to construct, from background extenders that have only one long generator, coarse models $M$ which either reach all finite levels of supercompactness or satisfy the following covering property at many $\kappa:\left(\beth_{\omega}(\kappa)\right)^{M} \geq\left(\kappa^{+}\right)^{V}$.

Fix an inaccessible cardinal $\delta$. Let $\mathcal{W}$ be defined as in Section 2, adapting the construction to allow long extender premice, but maintaining the same background condition, namely the condition in Definition 2.1, also for long extenders. In particular the backgrounds $F^{*}$ are always strictly short.

There are some fine structural modifications to the construction of AndrettaNeeman-Steel [1], for example we allow an additional coring operation, taking hulls of ranges of long extenders. But these are done in a way that preserves 
the properties of the resurrection maps mentioned in Section 2. Because of this, and since we maintain exactly the same certifiability condition even as we pass to long extenders, the iterability proof in Section 2 continues to apply, and $\mathcal{W}$ is weakly iterable. Of course the proof is restricted to non-overlapping trees, as defined above, because in Claim 2.3 it is essential that $\operatorname{crit}\left(F_{\epsilon}\right)<F_{\xi}\left(\operatorname{crit}\left(F_{\xi}\right)\right)$ where $\xi$ is the $<_{\mathcal{T}}$ predecessor of $\epsilon+1$. Fortunately, as we saw above, these trees suffice for comparison.

As in Section 2, the model $\mathcal{W}$ produced by the construction satisfies a maximality condition to the effect that certifiable extenders that can be placed on the sequence are indeed on the sequence:

Suppose $\kappa<\lambda<\delta$ are cardinals of $\mathcal{W}$, and $F$ is an extender (possibly long) over $\mathcal{W}$ mapping its domain cofinally into $\mathcal{W}\lceil\alpha$, with $\operatorname{crit}(F)=\kappa$ and $F(\operatorname{crit}(F))=\lambda$. Suppose that in $V_{\delta}$ there is an extender $F^{*}$ over $V$ and an embedding $h$ so that $\operatorname{crit}\left(F^{*}\right)=\kappa, F^{*}$ is $\eta$ strong for some inaccessible cardinal $\eta>\alpha$, $\operatorname{crit}(h) \geq \lambda$, and $h \circ F \subseteq i_{F^{*}}$ where $i_{F^{*}}$ is the ultrapower embedding of $V$ by $F^{*}$.

Suppose in addition that $\rho(\mathcal{W}\lceil\alpha ; F) \leq \lambda$ and that there is a parameter witnessing this which satisfies the constraints imposed in [7] to guarantee that it is moved correctly in comparisons.

Then $F$ is on the sequence of $\mathcal{W}$, indexed at $\alpha$.

This maximality condition has a clause on the projectum of $(\mathcal{W}\lceil\alpha ; F)$ that was not present in Lemma 2.4 (though in any case it holds automatically for non-long extenders). This is in line with the restriction on long extenders that was necessary for termination of the comparison process described above. We leave out the exact constraints imposed in [7], but note that they are satisfied in the situation we encounter in the argument that follows.

Suppose now that $\delta$ satisfies the assumption of Theorem 1.9 for $n=2$. Precisely, suppose that in $V^{\mathrm{Col}\left(\delta, \delta^{++}\right)}, \delta$ is weakly compact and for every $Z$ in the Woodin filter for $\delta$, the weak compactness of $\delta$ can be witnessed by partial measures concentrating on $Z$. We construct an extension of $\mathcal{W}$ in which $\delta$ is $+(2)$ subcompact.

Let $G$ be generic for $\operatorname{Col}\left(\delta, \delta^{++}\right)$. We work in $V[G]$. Let $U_{1}=U(\mathcal{W})$ and let $\mathcal{S}_{1}=\mathcal{S}(\mathcal{W})$. By Corollary 3.5 both belong to $V$. Let $\gamma_{1}=\operatorname{Ord} \cap \mathcal{S}_{1}$. Since $\mathcal{S}_{1}$ belongs to $V$ and has unboundedly many levels that project to $\delta, \gamma_{1} \leq\left(\delta^{+}\right)^{V}$.

By Claim 4.1, or more precisely the adaptation of this claim to the construction of $\mathcal{W}$ that allows also long extenders, $\operatorname{Cof}\left(\gamma_{1}\right) \geq \delta$. Hence by Lemma 3.2, $U\left(\mathcal{S}_{1}\right)$ and $\mathcal{S}\left(\mathcal{S}_{1}\right)$ are well defined. Let $\mathcal{S}_{2}=\mathcal{S}\left(\mathcal{S}_{1}\right)$ and let $\gamma_{2}=\operatorname{Ord} \cap \mathcal{S}_{2}$. By Corollary $3.5, \mathcal{S}_{2}$ belongs to $V$.

Since $\mathcal{S}_{2}$ has unboundedly many levels that project to $\gamma_{1}, \gamma_{2} \leq\left(\gamma_{1}^{+}\right)^{V} \leq$ $\left(\delta^{++}\right)^{V}$. Hence in $V[G], \mathcal{S}_{2}$ has size $\delta$. We can therefore fix an increasing continuous chain $\vec{H}=\left\langle H_{\xi} \mid \xi<\delta\right\rangle$ with $\left|H_{\xi}\right|<\delta$ and $\bigcup_{\xi<\delta} H_{\xi}=\mathcal{S}_{2}$. Using Lemma 3.6 for the chain $\left\langle H_{\xi} \cap \mathcal{S}_{1} \mid \xi<\delta\right\rangle$ we can further fix a club $C \subseteq \delta$ so that for every $\xi \in C, H_{\xi} \cap \delta=\xi$, and every strict initial segment of the transitive collapse of $H_{\xi} \cap \mathcal{S}_{1}$ is an initial segment of $\mathcal{W}$ below $\left(\xi^{+}\right)^{\mathcal{W}}$.

As a matter of notation, call a partial measure $\mu$ over $\delta$ suitable for $X$ if the domain of $\mu$ includes all sets of the form $\{\alpha<\delta \mid f(\alpha) \in g(\alpha)\}$ and $\{\alpha<\delta \mid$ 
$f(\alpha)=g(\alpha)\}$ for $f, g \in X \cup\left\{i d_{c} \mid c \in V_{\delta}\right\}$, where $i d_{c}$ is the function on $\delta$ taking constant value $c$. The ultrapower of $X$ by $\mu$ then makes sense. Let $i_{\mu}^{X}$ denote the ultrapower embedding.

Call $X$ nice if $X$ is transitive of size $\delta$, countably closed, and satisfies a large enough finite fragment of ZFC to give the instances elementarity of $i_{\mu}^{X}$ that we need in the arguments below.

For nice $X$ and suitable $\mu$, let $\mathcal{W}^{*}(X, \mu)$ denote $i_{\mu}^{X}(\mathcal{W})$.

Claim 5.2. For every nice $X \supseteq V_{\delta} \cup\{\vec{H}, C\}$ and every $\mu$ suitable for $X$, $\mathcal{W}^{*}(X, \mu)\left\lceil\left(\delta^{+}\right)^{\mathcal{W}^{*}(X, \mu)}\right.$ is equal to $\mathcal{S}_{1}$. In particular, $\left(\delta^{+}\right)^{\mathcal{W}^{*}(X, \mu)}=\gamma_{1}$.

Proof. Let $i$ denote $i_{\mu}^{X}$ and let $\mathcal{W}^{*}=\mathcal{W}^{*}(X, \mu)$. Since $\vec{H}$ is continuous, $i(\vec{H})_{\delta}$ is exactly equal to $i^{\prime \prime} \mathcal{S}_{2}$. Hence the transitive collapse of $i(\vec{H})_{\delta} \cap i\left(\mathcal{S}_{1}\right)$ is equal to $\mathcal{S}_{1}$. Through our use of Lemma 3.6 above and since $\delta \in i(C)$ it follows that every strict initial segment of $\mathcal{S}_{1}$ is an initial segment of $\mathcal{W}^{*}$.

To complete the proof of the claim, suppose for contradiction that $\mathcal{W}^{*} \uparrow\left(\delta^{+}\right)^{\mathcal{W}^{*}}$ extends $\mathcal{S}_{1}$ strictly. Then there is $\mathcal{M} \triangleleft \mathcal{W}^{*}$ which projects to $\delta$ and extends $\mathcal{S}_{1}$ strictly. $\mathcal{W}$ is weakly iterable in $V$, hence also in $V[G]$ since $G$ does not add any bounded subsets of $\delta$. It follows using the countable closure of $X$ (part of the definition of being nice) and the elementarity of $i$ that $\mathcal{W}^{*}$ is weakly iterable. So $\mathcal{M}$ belongs to $U_{1}$. But this contradicts the fact that $\mathcal{M}$ strictly extends $\mathcal{S}_{1}$. $\dashv$

For $X$ and $\mu$ as in Claim 5.2, all levels of $\mathcal{W}^{*}(X, \mu)$ that project to $\gamma_{1}=$ $\left(\delta^{+}\right)^{\mathcal{W}^{*}(X, \mu)}$ belong to $U\left(\mathcal{S}_{1}\right)$, and hence are initial segments of $\mathcal{S}_{2}$. In particular, $\left(\delta^{++}\right)^{\mathcal{W}^{*}(X, \mu)} \leq \gamma_{2}$.

Let $\hat{\gamma}_{2}$ be least so that for every $A \subseteq \delta$, there exists a nice $X \supseteq V_{\delta} \cup\{\vec{H}, C, A\}$ and a partial measure $\mu$ suitable for $X$ with $\mu(\operatorname{Strong}(A))=1$, so that $\hat{\gamma}_{2} \geq$ $\left(\delta^{++}\right)^{\mathcal{W}^{*}(X, \mu)} \cdot \gamma_{2}$ satisfies this condition, by the conclusion of the previous paragraph and since the existence of $X$ and $\mu$ as above is given by our assumption that, in $V[G]$, the weak compactness of $\delta$ can be witnessed by partial measures concentrating on any $Z$ in the Woodin filter for $\delta$. So $\hat{\gamma}_{2}$ is well defined, and $\hat{\gamma}_{2} \leq \gamma_{2}$. In particular $\left|\hat{\gamma}_{2}\right|=\delta$ in $V[G]$. Let $\hat{\mathcal{S}}_{2}=\mathcal{S}_{2} \uparrow \hat{\gamma}_{2}$.

Claim 5.3. For every $A \subseteq \delta$, there exists nice $X \supseteq V_{\delta} \cup\{\vec{H}, C, A\}$ and a partial measure $\mu$ suitable for $X$ with $\mu(\operatorname{Strong}(A))=1$, so that $\left(\delta^{++}\right)^{\mathcal{W}^{*}(X, \mu)}=\hat{\gamma}_{2}$ and $\mathcal{W}^{*}(X, \mu)\left\lceil\left(\delta^{++}\right)^{\mathcal{W}^{*}(X, \mu)}=\hat{\mathcal{S}}_{2}\right.$.

Proof. Fix $A$. For each $\xi<\hat{\gamma}_{2}$, let $A_{\xi}$ witness the failure of the condition defining $\hat{\gamma}_{2}$, at $\xi$. Using the fact that $\left|\hat{\gamma}_{2}\right|=\delta$ (working in $V[G]$ ), let $A^{*}$ be a subset of $\delta$ coding $\left\langle A_{\xi} \mid \xi<\hat{\gamma}_{2}\right\rangle$ and $A$. The precise coding does not matter. What is important is that $\operatorname{Strong}\left(A^{*}\right) \subseteq \operatorname{Strong}(A)$, for every $\xi<\hat{\gamma}_{2}$, every sufficiently large $\kappa \in \operatorname{Strong}\left(A^{*}\right)$ belongs to $\operatorname{Strong}\left(A_{\xi}\right)$, and any $X \supseteq V_{\delta} \cup\left\{A^{*}\right\}$ satisfying the fragment of ZFC required for being nice contains $\left\{A_{\xi} \mid \xi<\hat{\gamma}_{2}\right\} \cup$ $\{A\}$.

Let $X$ and $\mu$ be given by the definition of $\hat{\gamma}_{2}$ applied with $A^{*}$, so that $\hat{\gamma}_{2} \geq$ $\left(\delta^{++}\right) \mathcal{W}^{*}(X, \mu)$. By choice of $A_{\xi}$ and since $A^{*}$ codes $A_{\xi}$, for every $\xi<\hat{\gamma}_{2}, \xi \nsupseteq$ $\left(\delta^{++}\right)^{\mathcal{W}^{*}(X, \mu)}$. So it must be that $\hat{\gamma}_{2}=\left(\delta^{++}\right)^{\mathcal{W}^{*}(X, \mu)}$. Since all strict initial segments of $\mathcal{W}^{*}(X, \mu)\left\lceil\left(\delta^{++}\right)^{\mathcal{W}^{*}(X, \mu)}\right.$ are initial segments of $\mathcal{S}_{2}$, it follows that $\mathcal{W}^{*}(X, \mu)\left\lceil\left(\delta^{++}\right)^{\mathcal{W}^{*}(X, \mu)}=\mathcal{S}_{2}\left\lceil\hat{\gamma}_{2}=\hat{\mathcal{S}}_{2}\right.\right.$. 
Claim 5.4. $\hat{\gamma}_{2}$ is a cardinal of $L\left(\hat{\mathcal{S}}_{2}\right)$, and all bounded subsets of $\hat{\gamma}_{2}$ in $L\left(\hat{\mathcal{S}}_{2}\right)$ belong to $\hat{\mathcal{S}}_{2}$.

Proof. Working with the objects in the proof of Claim 5.3, we have that $\hat{\gamma}_{2}$ is a cardinal of $\mathcal{W}^{*}(X, \mu)$, and all bounded subsets of $\hat{\gamma}_{2}$ in $\mathcal{W}^{*}(X, \mu)$ belong to $\mathcal{W}^{*}(X, \mu)\left\lceil\hat{\gamma}_{2}=\hat{\mathcal{S}}_{2}\right.$. It is clear that $\mathcal{W}^{*}(X, \mu) \supseteq L_{\theta}\left(\mathcal{W}^{*}(X, \mu)\left\lceil\hat{\gamma}_{2}\right)=L_{\theta}\left(\hat{\mathcal{S}}_{2}\right)\right.$ for every $\theta \leq \operatorname{Ord} \cap \mathcal{W}^{*}(X, \mu)=i_{\mu}^{X}(\delta)$. In $\operatorname{Ult}_{0}(X, \mu), i_{\mu}^{X}(\delta)$ is a Woodin cardinal, hence a limit of measurable cardinals. So, in $\operatorname{Ult}_{0}(X, \mu)$, there is a measurable cardinal $\theta>\hat{\gamma}_{2}$ so that $\hat{\gamma}_{2}$ is a cardinal in $L_{\theta}\left(\hat{\mathcal{S}}_{2}\right)$, and all bounded subsets of $\hat{\gamma}_{2}$ in $L_{\theta}\left(\hat{\mathcal{S}}_{2}\right)$ belong to $\hat{\mathcal{S}}_{2}$. The claim follows by iterating a measure on $\theta$ that belongs to $\operatorname{Ult}_{0}(X, \mu)$ through the ordinals. The measure is fully iterable using its iterability in $\operatorname{Ult}_{0}(X, \mu)$ and the countable closure of $X$.

We proceed now to show that $\delta$ is $+(2)$ subcompact in $L\left(\hat{\mathcal{S}}_{2}\right)$. We do this by showing that enough extenders derived from anticollapse maps belong to $\hat{\mathcal{S}}_{2}$.

Let $\mathcal{N}_{\xi}$ be the transitive collapse of $H_{\xi} \cap \hat{\mathcal{S}}_{2}$. Let $\sigma_{\xi}: \mathcal{N}_{\xi} \rightarrow \hat{\mathcal{S}}_{2}$ be the anticollapse embedding. Let $\sigma_{\xi, \zeta}: \mathcal{N}_{\xi} \rightarrow \mathcal{N}_{\zeta}$ for $\xi<\zeta$ be the map $\sigma_{\zeta}^{-1} \circ \sigma_{\xi}$. Let $\vec{\sigma}$ denote $\left\langle\sigma_{\xi, \zeta} \mid \xi<\zeta<\delta\right\rangle$ and let $\overrightarrow{\mathcal{N}}$ denote $\left\langle\mathcal{N}_{\xi} \mid \xi<\delta\right\rangle$. Let $A \subseteq \delta$ code all these objects.

Let $X$ and $\mu$ be given by Claim 5.3. Then by the claim and since $i_{\mu}^{X}(\overrightarrow{\mathcal{N}})_{\delta}=\hat{\mathcal{S}}_{2}$, the set of $\kappa$ so that

I1. $\kappa$ is $<\delta$ strong relative to $A$, and

I2. $\mathcal{N}_{\kappa}=\mathcal{W} \uparrow\left(\kappa^{++}\right)^{\mathcal{W}}$

has $\mu$ measure 1 . Let $I$ denote this set.

For $\kappa<\tau$ both in $I, \operatorname{crit}\left(\sigma_{\kappa}\right)=\operatorname{crit}\left(\sigma_{\kappa, \tau}\right)=\kappa, \sigma_{\kappa}(\kappa)=\delta$, and $\sigma_{\kappa, \tau}(\kappa)=\tau$. Let $E_{\kappa, \tau}$ be the $(\kappa, \tau)$ extender derived from $\sigma_{\kappa, \tau}$. Precisely this is the restriction of $\sigma_{\kappa, \tau}$ to $\mathcal{W} \uparrow\left(\kappa^{+}\right)^{\mathcal{W}}$. Let $F_{\kappa, \tau}$ be the $\left(\left(\kappa^{+}\right)^{\mathcal{W}},\left(\tau^{+}\right)^{\mathcal{W}}\right)$ long extender derived from $\sigma_{\kappa, \tau}$. Viewed as an embedding this is exactly $\sigma_{\kappa, \tau}$, whose domain is $\mathcal{W} \uparrow\left(\kappa^{++}\right)^{\mathcal{W}}$. Similarly let $F_{\kappa}$ be the $\left(\left(\kappa^{+}\right)^{\mathcal{W}}, \gamma_{1}\right)$ long extender derived from $\sigma_{\kappa}$. Viewed as an embedding this is simply $\sigma_{\kappa}$ itself. We will show that, for appropriately chosen $\kappa<\tau, F_{\kappa, \tau}$ belongs to $\mathcal{W}$. This implies that $F_{\kappa, \tau}$ in fact belongs to $\mathcal{W} \uparrow\left(\tau^{++}\right)^{\mathcal{W}}=\mathcal{N}_{\tau}$. Applying $\sigma_{\tau}$ it follows that $F_{\kappa}$ belongs to $\hat{\mathcal{S}}_{2}$. We will obtain this for a stationary set of $\kappa<\delta$, thereby producing enough extenders in $L\left(\hat{\mathcal{S}}_{2}\right)$ to witness that $\delta$ is $+(2)$ subcompact.

We cannot expect to argue that $F_{\kappa, \tau}$ is on the sequence $\vec{E}^{\mathcal{W}}$; it would have to be indexed above $\left(\tau^{+}\right)^{\mathcal{W}}$, contradicting our requirement that active stages with top predicate $F$ project to $F(\operatorname{crit}(F))$. Instead we will show that a stretch of $F_{\kappa, \tau}$ is on the sequence, and that there is also a superstrong extender on the sequence that allows recovering $F_{\kappa, \tau}$ from that stretch.

Claim 5.5. Let $\tau<\lambda$ both belong to I. Then $E_{\tau, \lambda}$ is on the sequence of $\mathcal{W}$, indexed at $\sup \left(\sigma_{\tau, \lambda}{ }^{\prime \prime}\left(\tau^{+}\right)^{\mathcal{W}}\right)$.

Proof. This is similar to Claim 4.1. Let $\eta<\delta$ be an inaccessible cardinal above $\lambda$. Let $F^{*} \in V_{\delta}$ be an extender with critical point $\tau$, which is $\eta$ strong relative to $A$, hence also relative to $\overrightarrow{\mathcal{N}}$ and $\vec{\sigma}$. Let $i_{F^{*}}$ be the ultrapower embedding by $F^{*}$, and let $\tau^{*}=i_{F^{*}}(\tau)$. Let $h$ be the restriction of $i_{F^{*}}(\vec{\sigma})_{\lambda, \tau^{*}}$ to $\mathcal{N}_{\lambda}\left\lceil\left(\lambda^{+}\right)^{\mathcal{W}}\right.$. 
Then a computation as in the proof of Claim 4.1 shows that $h \circ E_{\tau, \lambda}=$ $i_{F^{*}} \uparrow\left(\mathcal{N}_{\tau}\left\lceil\left(\tau^{+}\right)^{\mathcal{W}}\right)\right.$. It follows using the maximality condition for $\mathcal{W}$ that $E_{\tau, \lambda}$ is on the sequence of $\mathcal{W}$, indexed at $\alpha=\sup \left(\sigma_{\tau, \lambda}{ }^{\prime \prime}\left(\tau^{+}\right)^{\mathcal{W}}\right)$.

Claim 5.6. Let $\kappa<\tau$ both belong to I. Then $F_{\kappa, \tau}$ belongs to $\mathcal{W}$, and therefore $F_{\kappa}$ belongs to $\hat{\mathcal{S}}_{2}$.

Proof. If $F_{\kappa, \tau} \in \mathcal{W}$ then in fact $F_{\kappa, \tau} \in \mathcal{W} \|\left(\tau^{++}\right)^{\mathcal{W}}=\mathcal{N}_{\tau}$, so $\sigma_{\tau}\left(F_{\kappa, \tau}\right) \in \hat{\mathcal{S}}_{2}$, and since $\sigma_{\tau}\left(F_{\kappa, \tau}\right)=\sigma_{\tau} \circ F_{\kappa, \tau}=F_{\kappa}$ it follows that $F_{\kappa} \in \hat{\mathcal{S}}_{2}$.

Suppose for contradiction that $F_{\kappa, \tau} \notin \mathcal{W}$. Fix $\lambda>\tau$ in $I$. Let $\eta<\delta$ be an inaccessible cardinal above $\lambda$. Let $F^{*} \in V_{\delta}$ be an extender with critical point $\kappa$, which is $\eta$ strong relative to $A$, hence also relative to $\overrightarrow{\mathcal{N}}$ and $\vec{\sigma}$. Let $i_{F^{*}}$ be the ultrapower embedding by $F^{*}$, and let $\kappa^{*}=i_{F^{*}}(\kappa)$. Let $h=i_{F^{*}}(\vec{\sigma})_{\lambda, \kappa^{*}}$.

A computation similar to that in Claim 4.1 shows that $h \circ F_{\kappa, \lambda}=i_{F^{*}}\left\lceil\mathcal{N}_{\kappa}\right.$. Now by the maximality condition for $\mathcal{W}$, it would follow that $F_{\kappa, \lambda}$ is on the sequence of $\mathcal{W}$, indexed at $\alpha=\sup \left(\sigma_{\kappa, \lambda}{ }^{\prime \prime}\left(\kappa^{++}\right)^{\mathcal{W}}\right)$, provided we can show that $\left(\mathcal{W}\left\lceil\alpha ; F_{\kappa, \lambda}\right)\right.$ projects to or below $\lambda$. (Since we are dealing with a long extender, this is not automatic.)

Notice that the restriction of $F_{\kappa, \tau}$ to subsets of $\left(\kappa^{+}\right)^{\mathcal{W}}$ is $\Sigma_{1}$ definable over $\left(\mathcal{W}\left\lceil\alpha ; F_{\kappa, \lambda}\right)\right.$, using the parameter $E_{\tau, \lambda}$, which by Claim 5.5 we know is an element of $\mathcal{W}$ and hence in fact an element of $\mathcal{W}\left\lceil\left(\lambda^{+}\right)^{\mathcal{W}} \subseteq \mathcal{W}\left\lceil\alpha\right.\right.$. The $\Sigma_{1}$ definition is simply that, for $x, y \in \mathcal{W}$ with $x \subseteq\left(\kappa^{+}\right)^{\mathcal{W}}$ and $y \subseteq\left(\tau^{+}\right)^{\mathcal{W}},\langle x, y\rangle \in F_{\kappa, \tau}$ iff $(\exists z)\left(\langle x, z\rangle \in F_{\kappa, \lambda}\right.$ and $y$ is the preimage of $z$ under $\left.E_{\tau, \lambda}\right)$. This equivalence is obtained using the following computation for $\nu<\left(\tau^{+}\right)^{\mathcal{W}}$ :

$$
\begin{aligned}
\nu \in F_{\kappa, \tau}(x) & \Longleftrightarrow \nu \in \sigma_{\kappa, \tau}(x) \\
& \Longleftrightarrow \sigma_{\tau, \lambda}(\nu) \in \sigma_{\tau, \lambda}\left(\sigma_{\kappa, \tau}(x)\right) \\
& \Longleftrightarrow \sigma_{\tau, \lambda}(\nu) \in \sigma_{\kappa, \lambda}(x) \\
& \Longleftrightarrow E_{\tau, \lambda}(\nu) \in F_{\kappa, \lambda}(x) .
\end{aligned}
$$

Recall that we assumed for contradiction that $F_{\kappa, \tau} \notin \mathcal{W}$. Since $F_{\kappa, \tau}$ is completely determined by its restriction to subsets of $\left(\kappa^{+}\right)^{\mathcal{W}}$ it follows that this restriction does not belong to $\mathcal{W}$. We saw above that the restriction is $\Sigma_{1}$ definable over $\left(\mathcal{W}\left\lceil\alpha ; F_{\kappa, \lambda}\right)\right.$ from the parameter $E_{\tau, \lambda}$. Since the restriction determines a new subset of $\left(\tau^{+}\right)^{\mathcal{W}}$ it follows that $\rho\left(\mathcal{W}\left\lceil\alpha ; F_{\kappa, \lambda}\right) \leq\left(\tau^{+}\right)^{\mathcal{W}}<\lambda\right.$.

Using the maximality principle for $\mathcal{W}$, we can now conclude that $F_{\kappa, \lambda}$ is on the sequence of $\mathcal{W}$, indexed at $\alpha$.

But then $F_{\kappa, \lambda}$ is an element of $\mathcal{W}$. Since $E_{\tau, \lambda}$ is also an element of $\mathcal{W}$, and since, as we saw above, $F_{\kappa, \tau}$ can be recovered from $F_{\kappa, \lambda}$ and $E_{\tau, \lambda}$, it follows that $F_{\kappa, \tau}$ is an element of $\mathcal{W}$.

Claim 5.7. $\delta$ is $+(2)$ subcompact in $L\left(\hat{\mathcal{S}}_{2}\right)$.

Proof. Let $B \subseteq \hat{\mathcal{S}}_{2}=L\left(\hat{\mathcal{S}}_{2}\right)\left\lceil\left(\delta^{++}\right)^{L\left(\hat{\mathcal{S}}_{2}\right)}\right.$. For each $\xi<\delta$, let $B_{\xi}$ be the preimage of $B$ under $\sigma_{\xi}$. For all $\xi$ in some club $D \subseteq \delta, \sigma_{\xi}$ is elementary as a map from $\left(\mathcal{N}_{\xi} ; \xi, B_{\xi}\right)$ into $\left(\hat{\mathcal{S}}_{2} ; \delta, B\right)$.

Modifying $X$ and $\mu$ if necessary, we may assume that $D \in X$. (We obtained $X$ and $\mu$ through an application of Claim 5.3. The claim produces $X \supseteq V_{\delta} \cup$ $\{\vec{H}, C, A\}$ for any given $A \subseteq \delta$, and we may revise $A$ to code $D$.) Since all 
clubs that belong to $X$ are given measure 1 by $\mu$, and since the set $I$ defined by conditions (I1) and (I2) above is also given measure $1, D \cap I$ is not empty. Fix $\kappa \in D \cap I$. By Claim 5.6, $F_{\kappa}=\sigma_{\kappa}$ belongs to $\hat{\mathcal{S}}_{2}$. By condition (I2), $\mathcal{N}_{\kappa}=\mathcal{W} \uparrow\left(\kappa^{++}\right)^{\mathcal{W}}$. So, in $L\left(\hat{\mathcal{S}}_{2}\right)$, there is an elementary embedding, namely $\sigma_{\kappa}$, of $\left(\mathcal{W} \uparrow\left(\kappa^{++}\right)^{\mathcal{W}} ; \kappa, B_{\kappa}\right)$ into $\left(\hat{\mathcal{S}}_{2} ; \delta, B\right)$.

Under the restriction of the assumption of Theorem 1.9 to $n=2$ we saw above how to obtain a class inner model where $\delta$ is $+(2)$ subcompact. Using similar arguments for larger $n<\omega$ one can continue to identify "stable" values for $\left(\delta^{+(n)}\right)^{\mathcal{W}^{*}(X, \mu)}$ and $\mathcal{W}^{*}(X, \mu) \uparrow\left(\delta^{+(n)}\right)^{\mathcal{W}^{*}(X, \mu)}$, and show that enough of the anticollapse embeddings belong to these models to witness that $\delta$ is $+(n)$ subcompact. The proof that enough anticollapse embeddings belong to the model is by induction on $n$, where at every stage maps obtained in the previous stage are used as parameters that allow recovering the current maps from their stretches. As in Claim 5.6, the stretch satisfies the low projectum requirement in case the original map is not in $\mathcal{W}$. For details we refer the reader to [7].

\section{REFERENCES}

[1] Alessandro Andretta, Itay Neeman, and John Steel, The domestic levels of $K^{c}$ are iterable, Israel J. Math., vol. 125 (2001), pp. 157-201.

[2] Ronald Jensen, Ernest Schimmerling, Ralf Schindler, and John Steel, Stacking mice, J. Symbolic Logic, vol. 74 (2009), no. 1, pp. 315-335.

[3] Kyriakos Kypriotakis and Martin Zeman, A characterization of $\square\left(\kappa^{+}\right)$in extender models, Arch. Math. Logic, vol. 52 (2013), no. 1-2, pp. 67-90.

[4] D. A. Martin and J. R. Steel, Iteration trees, J. Amer. Math. Soc., vol. 7 (1994), no. 1 , pp. $1-73$.

[5] William Mitchell and Ralf Schindler, A universal extender model without large cardinals in V, J. Symbolic Logic, vol. 69 (2004), no. 2, pp. 371-386.

[6] William J. Mitchell and John R. Steel, Fine structure and iteration trees, Lecture Notes in Logic, vol. 3, Springer-Verlag, Berlin, 1994.

[7] Itay Neeman and John Steel, Equiconsistencies at finite gap subcompactness, To appear.

[8] — , A weak Dodd-Jensen lemma, J. Symbolic Logic, vol. 64 (1999), no. 3, pp. 12851294.

[9] Grigor Sargsyan, A tale of hybrid mice, To appear in the Memoirs of the American Mathematical Society.

[10] Ernest Schimmerling, Coherent sequences and threads, Adv. Math., vol. 216 (2007), no. 1 , pp. $89-117$.

[11] Ernest Schimmerling and Martin Zeman, Square in core models, Bull. Symbolic Logic, vol. 7 (2001), no. 3, pp. 305-314.

[12] — Characterization of $\square_{\kappa}$ in core models, J. Math. Log., vol. 4 (2004), no. 1, pp. $1-72$. 737.

[13] J. R. Steel, Local $K^{c}$ constructions, J. Symbolic Logic, vol. 72 (2007), no. 3, pp. 721-

[14] John R. Steel, Iterations with long extenders, (2002), Notes taken by Oliver Deiser, available at http://math.berkeley.edu/ steel/papers/longext.kappaplus.ps

[15] — Derived models associated to mice, Computational prospects of infinity. Part I. Tutorials, Lect. Notes Ser. Inst. Math. Sci. Natl. Univ. Singap., vol. 14, World Sci. Publ., Hackensack, NJ, 2008, pp. 105-193.

[16] W. Hugh Woodin, The fine structure of suitable extender models I, In preparation.

[17] — Suitable extender models I, J. Math. Log., vol. 10 (2010), no. 1-2, pp. 101339. 
[18] - Suitable extender models II: beyond w-huge, J. Math. Log., vol. 11 (2011), no. 2 , pp. $115-436$.

[19] Martin Zeman, Inner models and large cardinals, de Gruyter Series in Logic and its Applications, vol. 5, Walter de Gruyter \& Co., Berlin, 2002.

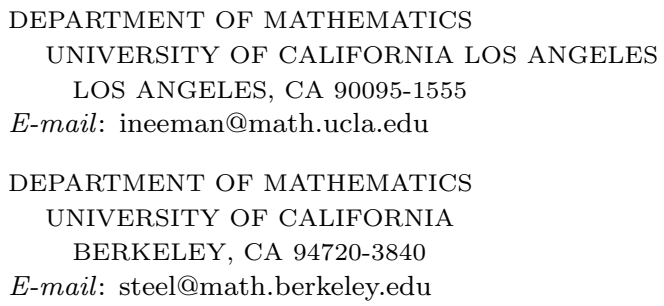

E-mail: steel@math.berkeley.edu 